\title{
Physical functional performance and prognosis in patients with heart failure: a systematic review and meta-analysis
}

Iván José Fuentes-Abolafio ${ }^{\text {', Brendon Stubbs 2,3,4 , Luis Miguel Pérez-Belmonte }}{ }^{5,6,7}$, María Rosa Bernal-López ${ }^{5,8}$, Ricardo Gómez-Huelgas ${ }^{5,8}$ and Antonio Ignacio Cuesta-Vargas ${ }^{1, *^{*}}$ (i)

\begin{abstract}
Background: Patients with Heart Failure (HF) show impaired functional capacities which have been related to their prognosis. Moreover, physical functional performance in functional tests has also been related to the prognosis in patients with HF. Thus, it would be useful to investigate how physical functional performance in functional tests could determine the prognosis in patients with HF, because HF is the leading cause of hospital admissions for people older than 65 years old. This systematic review and meta-analysis aims to summarise and synthesise the evidence published about the relationship between physical functional performance and prognosis in patients with $H F$, as well as assess the risk of bias of included studies and the level of evidence per outcome.

Methods: Major electronic databases, such as PubMed, AMED, CINAHL, EMBASE, PEDro, Web of Science, were searched from inception to March 2020 for observational longitudinal cohort studies (prospective or retrospective) examining the relationship between physical functional performance and prognosis in patients with HF.

Results: 44 observational longitudinal cohort studies with a total of 22,598 patients with HF were included. 26 included studies reported a low risk of bias, and 17 included studies showed a moderate risk of bias. Patients with poor physical functional performance in the Six Minute Walking Test (6MWT), in the Short Physical Performance Battery (SPPB) and in the Gait Speed Test showed worse prognosis in terms of larger risk of hospitalisation or mortality than patients with good physical functional performance. However, there was a lack of homogeneity regarding which cut-off points should be used to stratify patients with poor physical functional performance from patients with good physical functional performance.

Conclusion: The review includes a large number of studies which show a strong relationship between physical functional performance and prognosis in patients with HF. Most of the included studies reported a low risk of bias, and GRADE criteria showed a low and a moderate level of evidence per outcome.
\end{abstract}

Keywords: Functional tests, Heart failure, Hospitalisation, Mortality, Physical functional performance, Prognosis

\footnotetext{
* Correspondence: acuesta@uma.es

'Department of Physiotherapy, Faculty of Health Science, University of

Malaga, The Institute of Biomedical Research in Malaga (IBIMA), Clinimetric

Group FE-14, Malaga, Spain

${ }^{9}$ School of Clinical Sciences, Faculty of Health at the Queensland University

of Technology, Brisbane, Queensland, Australia

Full list of author information is available at the end of the article
}

(c) The Author(s). 2020 Open Access This article is licensed under a Creative Commons Attribution 4.0 International License, which permits use, sharing, adaptation, distribution and reproduction in any medium or format, as long as you give appropriate credit to the original author(s) and the source, provide a link to the Creative Commons licence, and indicate if changes were made. The images or other third party material in this article are included in the article's Creative Commons licence, unless indicated otherwise in a credit line to the material. If material is not included in the article's Creative Commons licence and your intended use is not permitted by statutory regulation or exceeds the permitted use, you will need to obtain permission directly from the copyright holder. To view a copy of this licence, visit http://creativecommons.org/licenses/by/4.0/ The Creative Commons Public Domain Dedication waiver (http://creativecommons.org/publicdomain/zero/1.0/) applies to the data made available in this article, unless otherwise stated in a credit line to the data. 


\section{Background}

Cardiovascular diseases continue to be the leading cause of disability-adjusted life-years (DALYs) due to noncommunicable diseases and the leading cause of death [1-3]. Within cardiovascular diseases, Heart Failure (HF) is the only cardiovascular disease which is increasing in incidence and prevalence due to the aging of the world population, because its prevalence increases with age [4-8]. In addition, heart failure constitutes the most important hospital diagnosis in older adults, is the leading cause of hospital admissions for people older than 65 years old and contributes to the increase of medical care costs [5-9].

Heart Failure is characterised by a weak myocardium with decreased cardiac output that is unable to meet the body metabolic demands [4-6, 8, 10-12]. There are several functional symptoms that appear in patients with $\mathrm{HF}$, such as reduced aerobic capacity, decreased muscle strength, low weekly physical activity and exercise intolerance, which are accompanied by fatigue and dyspnea symptoms [12-17]. Furthermore, patients with HF show impaired functional capacities, experience a declined ability to carry out their activities of daily living and suffer a reduced quality of life [12, 14, 17]. It has also been reported that patients with chronic HF show a slower gait speed than healthy subjects of the same age [18]. The maximal aerobic capacity has been inversely correlated to the severity of HF and has been directly correlated to the prognosis and the life expectancy [14, $19,20]$. Similarly, the lower extremities muscle mass and muscle strength have also been related to long-term survival in patients with $\operatorname{HF}[14,21]$.

Some functional tests have been used to predict prognosis in patients with HF. Thus, the 6-min walk test (6MWT) has been proposed as a simple, inexpensive, safe and reproducible exercise test to assess functional capacity in patients with HF, which could also predict the prognosis of patients with HF based on distance walked [12, 22-24]. The Short Physical Performance Battery (SPPB) provides a useful and indirect measure of muscle functional capacity [12]. Moreover, the SPPB and the Timed Up and Go test (TUG) could be used to assess physical or functional frailty in patients with HF, which has been associated with an increased risk of hospitalisation and mortality in chronic heart failure [25, 26]. The utility of Gait Speed has also been shown to predict functional independence loss, cardiovascular disease, hospitalisation, and mortality in older adults [27-31]. The 6-MWT measures the distance which patients can walk during $6 \mathrm{~min}$ [32]. The test is usually conducted in a closed corridor of $30 \mathrm{~m}$ where two marks are placed on the ground at a distance of $30 \mathrm{~m}$, and patients walk from one end to the other, during $6 \mathrm{~min}$ [32]. The SPPB includes 3 tests: balance (feet together, semitándem and tandem during $10 \mathrm{~s}$ each), gait speed ( $4 \mathrm{~m})$ and standing up and sitting on a chair 5 times. Each test is scored from 0 (worst performance) to 4 (best performance). The total score for the whole battery that is the addition of the 3 tests and ranges from 0 to 12 [33]. In the TUG test patients are sat down in a chair, and at the order to "go", they stand up from the chair, walk $3 \mathrm{~m}$ until a reach a line that is on the floor. Then, patients should turn, return to the chair walking and sit again [34].

Hence, it would be necessary to conduct a synthesis of evidence that explores the relationship between the physical functional performance in functional tests and the prognosis in patients with HF. A systematic review may permit the formation of firm conclusions through an exhaustive synthesis of data [35]. Thus, the aim of this study was to answer the following PECOS ( $\mathrm{P}$, participant; E, exposure; C, comparator; O, outcome; S, study design) question through a systematic review of the literature on observational longitudinal cohort studies (prospective or retrospective) (S): Do older patients with HF (P), who have poor physical functional performance in some functional tests, such as 6-MWT, SPPB, TUG or Gait Speed (E), show a worse prognosis (O) than those patients with good physical functional performance $(C)$ ?

\section{Methods}

The Systematic Review and Meta-analysis was conducted in accordance with the Preferred Reporting Items for Systematic Reviews and Meta-Analyses (PRISMA) statement [36]. The systematic review protocol was registered at the International Prospective Register of Systematic Reviews (PROSPERO: CRD42020177427).

\section{Data sources and search strategy}

Two independent reviewers (IJF-A and AIC-V) conducted a systematic search using relevant search terms that were developed from Medical Subject Headings $(\mathrm{MeSH})$ and keywords from other similar studies from inception to March, 24th 2020 using optimised search strategies in the following electronic databases: PubMed, AMED, CINAHL, EMBASE, PEDro, Web of Science (Additional file 1). A manual search of relevant eligible studies, to select any studies missed during the electronic search, was also conducted using cross-references identified in the reference lists within both original and review articles. The grey literature databases, such as New York Academy of Medicine Grey Literature Report, Open Grey and Google Scholar [37] were examined to identify any relevant unpublished data. References were exported, and duplicates were removed using the Mendeley desktop V.1.19.2 citation management software. 


\section{Eligibility criteria}

The aforementioned PECOS framework was followed to determine which studies were included in the present systematic review and meta-analysis. Each study had to meet the following inclusion criteria:

1. Observational longitudinal cohort studies (prospective or retrospective)(S) examining whether older patients with HF (P), who have a poor physical functional performance in some functional tests, such as 6-MWT, SPPB, TUG or Gait Speed $(\mathrm{E})$, show worse prognosis, assessed as larger risk of hospitalisation or mortality, (O) than those patients with good physical functional performance (C).

2. No restriction was applied on the participants' age, ethnicity, gender, HF diagnosis or on the New York Heart Association (NYHA) scale score.

3. No restriction was applied on the language.

4. Studies recruiting participants from any setting (general population, primary or secondary care).

5. Studies providing Odds Ratio (OR) or Hazard Ratio (HR) data.

The exclusion criteria were as follows:

1. All studies that did not include an observational longitudinal cohort design (e.g cross-sectional studies, randomised controlled trials).

2. Studies exploring the prognosis value of functional tests in patients with other cardiovascular diseases different from HF.

3. Studies examining the relationship between physical functional performance in functional tests and other outcomes different from mortality or hospitalisation.

4. Studies investigating the prognosis value of physical activity assessed as daily activity, exercise time per week or physical activity scales.

\section{Study selection}

Two independent reviewers (IJF-A and AIC-V) carried out the screening of titles and abstracts to detect potentially relevant records and also excluded those documents that were not original papers. The same reviewers conducted the screening of those articles that met all inclusion criteria. A short checklist was carried out and followed in order to select the relevant studies (Additional file 2). In case of disagreements, the articles were always included.

\section{Data extraction}

Two independent reviewers (IJF-A and AIC-V) identified the following relevant data from each study: study details (first author and year of publication), region, setting, study design, sample size, functional tests with their cutoff points and characteristics of participants (mean age, \%males), HF diagnosis, follow-up, outcome and main results. When necessary, an email was sent to the original authors to try to get OR or HR data that was not included in their original articles.

\section{Quality assessment}

The same two reviewers (IJF-A and AIC-V) assessed the risk of bias of the included observational longitudinal cohort studies using the Newcastle Ottawa Scale (NOS) [38]. The NOS has been decribed as a reliable and valid tool for assessing the quality of observational longitudinal cohort studies [38, 39].

\section{Data synthesis and analysis}

To assess the overall quality and the strength of the evidence per outcome, the Grading of Recommendations Assessment, Development and Evaluation (GRADE) approach was used [40, 41]. Two researchers (IJF-A and AIC-V) judged whether these factors were present for each outcome reported at least in two studies. Metaanalysis was conducted for each outcome reported in two or more studies, as long as studies assessed the same outcome with the same functional test and the same measurement unit, that is, HR or OR. Outcomes not included in the meta-analysis were reported using a descriptive quantitative analysis. Thus, the most relevant summary measure with the 95\% Confidence Interval (95\% CI) for each study was provided. The most relevant summary measure with its $95 \% \mathrm{CI}$ was extracted of adjusted multivariate models when it was possible. In each meta-analysis it was decided to use the inverse variance as statistical method, fixed effects as analysis model and the HR or OR as effect measures. Heterogeneity was assessed using $\mathrm{I}^{2}$ statistic $[42,43]$. Values of $>25 \%$ is considered as low heterogeneity, $>50 \%$ moderate heterogeneity, and $>75 \%$ high heterogeneity [42, 43]. When heterogeneity was moderate or high, random effects were used as analysis model. Moreover, when metaanalyses included patients with HF with reduced (HFrEF) and preserved (HFpEF) ejection fraction or meta-analyses revealed high heterogeneity, as long as the outcome was reported by three or more studies, sensitivity analyses were conducted including studies dealing only with patients with HFrEF because the inclusion of patients with different ejection fraction could be a source of heterogeneity or could bias the results. The mean effect sizes, 95\% CI, and $\mathrm{I}^{2}$ were calculated for each outcome and used to create forest plots for visualization of each meta-analysis using the Review Manager (RevMan) version 5.3 [44]. 


\section{Results}

\section{Characteristics of included studies}

A total of 3881 citations were identified through electronic databases, with 263 additional studies identified through Grey Literature Sources and 14 studies identified through manual search. One thousand six hundred seventy-one titles and abstracts were screened and 110 original papers were assessed. The number of studies retrieved from each database and the number of studies excluded in each screening phase are shown in Fig. 1. The full reference of excluded studies in the second stage $(n=66)$ is reported in Additional file 3 . The conflict of interest of included studies is shown in Additional file 4 . Of these, 44 observational longitudinal cohort studies (prospective or retrospective) with a total of 22,598 patients with HF were included. Twenty of the included studies $(45.45 \%)$ reported only patients with HFrEF. Twenty one of the included studies (47.72\%) showed patients with HFrEF and HFpEF. The 6MWT was the most used test $(n=33)$ followed by the Gait Speed test $(n=8)$ and the SPPB $(n=4)$. The characteristics of the included observational longitudinal cohort studies are reported in Table 1.

\section{Meta-analyses}

The outcomes assessed by each study, as well as the main results, the risk of bias summary and the GRADE summary are shown in Table 2. Forest plots and effect sizes of each meta-analysis can also be seen in Additional file 5 .

Patients with HFrEF, HFpEF and acute HF who showed a poor physical functional performance in the $6 \mathrm{MWT}$ reported a larger risk of All-Cause of Mortality $[\mathrm{HR}=2.2995 \% \mathrm{CI}(1.86-2.82), p<0.001]$ than those patients who showed a good physical functional performance (Fig. 2a). Moreover, patients with HFrEF who

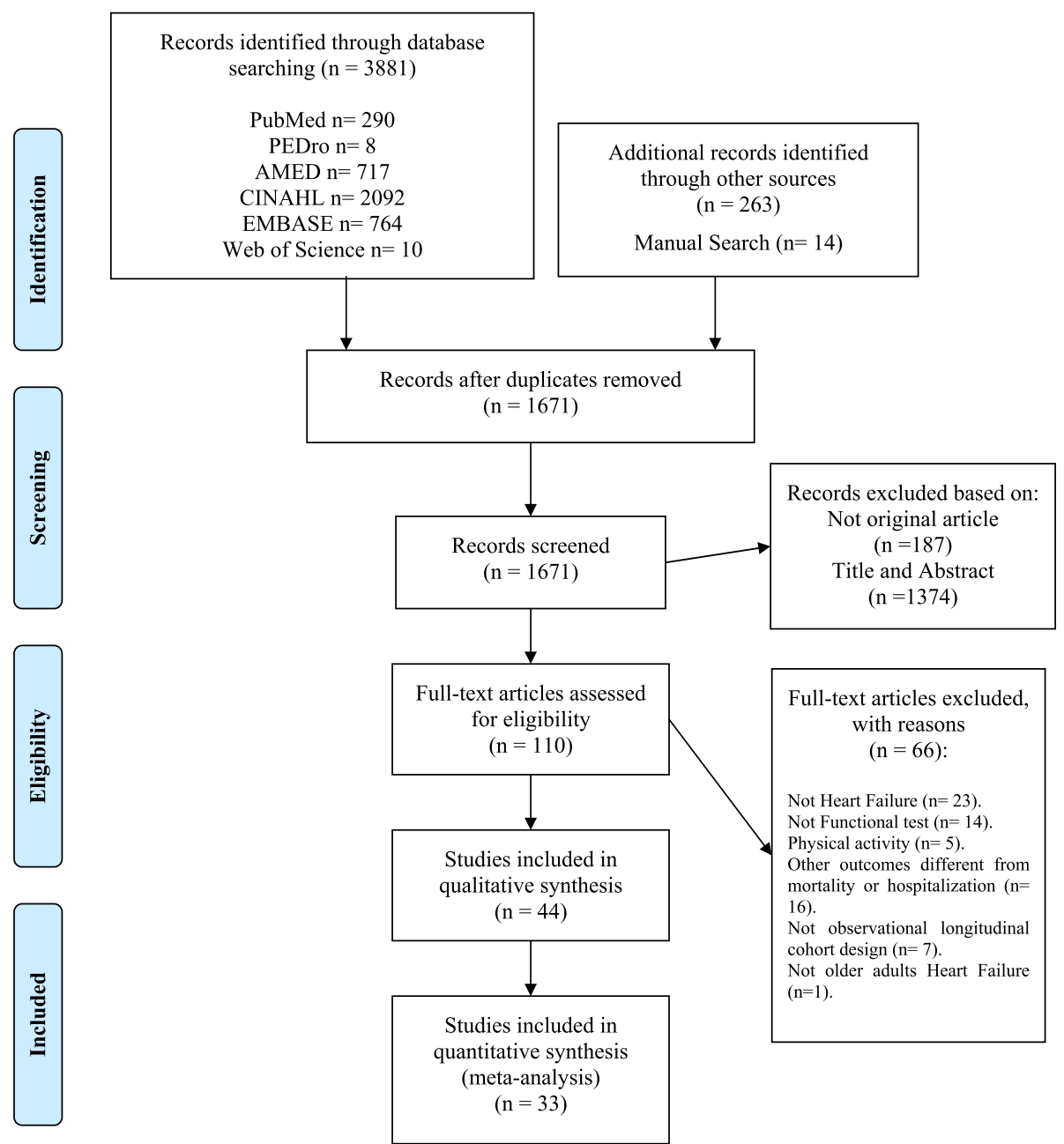

Fig. 1 Flow-Diagram. PRISMA 2009. From: Moher D, Liberati A, Tetzlaff J, Altman DG, The PRISMA Group (2009). Preferred Reporting Items for Systematic Reviews and Meta-Analyses: The PRISMA Statement. PLoS Med 6(6): e1000097. doi:https://doi.org/10.1371/journal.pmed1000097. For more information, visit www.prisma-statement.org 
Table 1 Characteristics of included studies

\begin{tabular}{|c|c|c|c|c|c|}
\hline $\begin{array}{l}\text { Study (first author and } \\
\text { year) }\end{array}$ & Region & Setting & Design & $\begin{array}{l}\text { Study Characteristics: Groups, } \\
\text { Sample Size (\%Male), Age }\end{array}$ & $\begin{array}{l}\text { Heart Failure } \\
\text { Diagnosis }\end{array}$ \\
\hline \multicolumn{6}{|c|}{ Six Minutes Walking Test (6MWT) } \\
\hline $\begin{array}{l}\text { Brenyo et al. [45], } \\
2012 \text {. }\end{array}$ & $\begin{array}{l}\text { United States, } \\
\text { Canada, and } \\
\text { Europe }\end{array}$ & $\begin{array}{l}\text { Clinical Care Setting } \\
\text { (110 Secondary Care } \\
\text { Centres) }\end{array}$ & Retrospective & $\begin{array}{l}\text { High Performance. } \\
>350 \mathrm{~m}: n=1021 \text { ( } 82 \%) .62 .5 \pm \\
10.5 \text { years. } \\
\text { Low Performance. } \\
\leq 350 \mathrm{m:} n=744(66.4 \%) .66 .8 \pm \\
10.7 \text { years. }\end{array}$ & $\begin{array}{l}\text { HFrEF } \\
\text { LVEF <30\% } \\
(29 \pm 3 \%)\end{array}$ \\
\hline $\begin{array}{l}\text { Ferreira et al. [46], } \\
2019\end{array}$ & $\begin{array}{l}11 \text { European } \\
\text { Countries }\end{array}$ & $\begin{array}{l}\text { Clinical Care Setting } \\
\text { (69 Secondary Care Centres) }\end{array}$ & Prospective & $\begin{array}{l}\text { High Performance. } \\
\text { > } 360 \mathrm{~m}: n=537 \text { ( } 86.6 \%) .62 \pm \\
11.0 \text { years. } \\
\text { Middle Performance. } \\
241-360 \mathrm{~m}: n=586(77.3 \%) .67 \pm \\
12.0 \text { years. } \\
\text { Low Performance. } \\
\leq 240 \mathrm{~m}: n=591(63 \%) .73 \pm \\
10.0 \text { years. }\end{array}$ & $\begin{array}{l}\text { HFrEF } \\
\text { LVEF }=30 \%(25- \\
38 \%)\end{array}$ \\
\hline $\begin{array}{l}\text { Wegrzynowska- } \\
\text { Teodorczyk et al. [47], } \\
2013 .\end{array}$ & Poland & $\begin{array}{l}\text { Clinical Care Setting } \\
\text { (Secondary Care) }\end{array}$ & Prospective & $\begin{array}{l}\text { All: } n=243(100 \%) .60 \pm 11.0 \\
\text { years. } \\
\text { High Performance. } \\
>468 \mathrm{~m} \text {. NS. } \\
\text { Low Performance. } \\
\leq 468 \mathrm{~m} . \text { NS. }\end{array}$ & $\begin{array}{l}\text { HFrEF } \\
\text { LVEF } \leq 45 \%(29 \pm \\
8 \%)\end{array}$ \\
\hline Bittner et al. [48], 1993. & $\begin{array}{l}\text { United States, } \\
\text { Canada, and } \\
\text { Belgium }\end{array}$ & $\begin{array}{l}\text { Clinical Care Setting ( } 20 \\
\text { Tertiary Care Hospitals) }\end{array}$ & Prospective & $\begin{array}{l}\text { All: } n=898 \text { (78\%). } 59 \pm 12.0 \\
\text { years. } \\
\text { High Performance. } \\
\geq 450: n=201 \text {. NS. } \\
\text { Middle Performance. } \\
\text { 375-450: } n=215 \text {. NS. } \\
\text { Low Performance. } \\
\text { 300-375: } n=241 \text {. NS. } \\
\text { Very Low Performance. } \\
<300: n=176 \text {. NS. }\end{array}$ & $\begin{array}{l}\text { Congestive HFrEF } \\
\mathrm{LVEF} \leq 45 \%\end{array}$ \\
\hline Arslan et al. [49], 2007. & Turkey & Not Reported & Prospective & $\begin{array}{l}\text { All: } n=43 \text { (86\%). } 62 \pm 10.0 \text { years. } \\
\text { High Performance. } \\
>300 \mathrm{~m} \text {. NS. } \\
\text { Low Performance. } \\
\leq 300 \mathrm{~m} \text {. NS. }\end{array}$ & $\begin{array}{l}\text { HFrEF } \\
\text { LVEF } \leq 40 \%(0.35 \pm \\
0.06 \%)\end{array}$ \\
\hline Lee et al. [50], 2006. & Singapore (Asian) & $\begin{array}{l}\text { Clinical Care Setting (Primary } \\
\text { and Secondary Care) }\end{array}$ & Prospective & $\begin{array}{l}\text { All: } n=668(67.4 \%) .66 .1 \pm 12.3 \\
\text { years. } \\
\text { High Performance. } \\
>370 \mathrm{~m}: n=87 . \mathrm{NS} \text {. } \\
\text { Middle Performance. } \\
311-370 \mathrm{~m}: n=84 . \mathrm{NS} \text {. } \\
\text { Low Performance. } \\
\text { 231-310 m: } n=87 . \mathrm{NS} \text {. } \\
\text { Very Low Performance. } \\
\text { 75-230 m: } n=128 . \mathrm{NS} \text {. }\end{array}$ & $\begin{array}{l}\text { HFrEF } \\
\text { LVEF }<40 \%\end{array}$ \\
\hline Curtis et al. [51], 2004. & $\begin{array}{l}\text { United States and } \\
\text { Canada }\end{array}$ & $\begin{array}{l}\text { Clinical Care Setting (39 } \\
\text { Secondary Care Centres) }\end{array}$ & Prospective & $\begin{array}{l}\text { High Performance. } \\
>400 \mathrm{~m}: n=131 \text { (91.6\%). } 60.0 \pm \\
11.0 \text { years. } \\
\text { Middle Performance. } \\
301-400 \mathrm{~m}: n=210 \text { (76.7\%). } \\
63.4 \pm 10.8 \text { years. } \\
\text { Low Performance. } \\
201-300 \mathrm{~m}: n=118(61.9 \%) . \\
66.8 \pm 10.4 \text { years. } \\
\text { Very Low Performance. } \\
\leq 200 \mathrm{~m}: n=82(54.9 \%) .70 .9 \pm \\
12.8 \text { years }\end{array}$ & $\begin{array}{l}\text { HFrEF and HFpEF } \\
\text { LVEF }<45 \% \text { (HFrEF) } \\
\text { LVEF }>45 \% \\
\text { (HFpEF) }\end{array}$ \\
\hline Ingle et al. [52], 2014. & United Kingdom & Not Reported & Prospective & $\begin{array}{l}\text { All: } n=1667(75 \%) .72(65-77) \\
\text { years. } \\
\text { High Performance. } \\
>360 \mathrm{~m}: n=\text { NS. } 64.9 \pm 10.6\end{array}$ & $\begin{array}{l}\text { HFrEF } \\
\text { LVEF }<45 \%\end{array}$ \\
\hline
\end{tabular}


Table 1 Characteristics of included studies (Continued)

\begin{tabular}{|c|c|c|c|c|c|}
\hline $\begin{array}{l}\text { Study (first author and } \\
\text { year) }\end{array}$ & Region & Setting & Design & $\begin{array}{l}\text { Study Characteristics: Groups, } \\
\text { Sample Size (\%Male), Age }\end{array}$ & $\begin{array}{l}\text { Heart Failure } \\
\text { Diagnosis }\end{array}$ \\
\hline & & & & $\begin{array}{l}\text { years. } \\
\text { Middle Performance. } \\
241-360 \mathrm{~m}: n=\mathrm{NS} .71 .3 \pm 8.8 \\
\text { years. } \\
\text { Low Performance. } \\
46-240 \mathrm{~m}: n=\mathrm{NS} .72 .9 \pm 9.6 \\
\text { years. } \\
\text { Very Low Performance. } \\
\leq 45 \mathrm{~m}: n=\text { NS. } 72.4 \pm 10.6 \text { years. }\end{array}$ & \\
\hline $\begin{array}{l}\text { Alahdab et al. [53], } \\
2009 .\end{array}$ & USA & $\begin{array}{l}\text { Clinical Care Setting (Tertiary } \\
\text { Care Hospital) }\end{array}$ & Prospective & $\begin{array}{l}\text { High Performance. } \\
>200 \mathrm{~m}: n=103 \text { ( } 75.7 \%) .50 .4 \pm \\
12.2 \text { years. } \\
\text { Low Performance. } \\
\leq 200 \mathrm{m:} n=95 \text { ( } 49.5 \%) .59 .4 \pm \\
12.2 \text { years. }\end{array}$ & $\begin{array}{l}\text { Acute } \\
\text { Decompensated } \\
\text { HFrEF and HFpEF } \\
\text { LVEF } \leq 40 \% \text { (HFrEF) } \\
\text { LVEF > 40\% } \\
\text { (HFpEF) }\end{array}$ \\
\hline $\begin{array}{l}\text { Mangla et al. [54], } \\
2013 \text {. }\end{array}$ & USA & $\begin{array}{l}\text { Clinical Care Setting } \\
\text { (Secondary Care) }\end{array}$ & Prospective & $\begin{array}{l}\text { All: } n=900(53 \%) .63 .6 \text { years. } \\
\text { High Performance. } \\
>189 \text { m. NS. } \\
\text { Low Performance. } \\
\leq 189 \text { m. NS. }\end{array}$ & $\begin{array}{l}\text { HFpEF and HFrEF } \\
\text { LVEF } \leq 40 \% \text { (HFrEF) } \\
\text { LVEF }>40 \% \\
\text { (HFpEF) }\end{array}$ \\
\hline Hasin et al. [55], 2012. & USA & $\begin{array}{l}\text { Clinical Care Setting } \\
\text { (Secondary Care) }\end{array}$ & Retrospective & $\begin{array}{l}\text { High Performance. } \\
\geq 300 \mathrm{~m}: n=45 \text { (87\%). } 65 \text { (53- } \\
69) \text { years. } \\
\text { Low Performance. } \\
<300 \mathrm{m:} n=20 \text { (75\%). } 68 \text { (59- } \\
74 \text { ) years. }\end{array}$ & $\begin{array}{l}\text { HFrEF } \\
\text { LVEF }<40 \%(20- \\
31 \%)\end{array}$ \\
\hline $\begin{array}{l}\text { Passantino et al. [56], } \\
2006 .\end{array}$ & Italy & $\begin{array}{l}\text { Clinical Care Setting } \\
\text { (Secondary Care) }\end{array}$ & Prospective & $\begin{array}{l}\text { All: } \mathrm{n}: 476 \text { (79\%). } 63.6 \pm 11.9 \\
\text { years. } \\
\text { High Performance. } \\
\geq 300 \mathrm{m:} n=301 . \mathrm{NS} \text {. } \\
\text { Low Performance. } \\
<300 \mathrm{~m}: n=175 . \mathrm{NS} \text {. }\end{array}$ & $\begin{array}{l}\text { HFrEF } \\
\text { LVEF < 40\% (29.8 } \pm \\
9.7)\end{array}$ \\
\hline $\begin{array}{l}\text { Howie-Esquivel et al. } \\
\text { [57], 2008. }\end{array}$ & USA & $\begin{array}{l}\text { An Academic } \\
\text { Medical Centre }\end{array}$ & Prospective & $\begin{array}{l}\text { High Performance. } \\
>200 \mathrm{~m}: n=21 \text { (73.3\%). } 61.7 \pm \\
17.3 \text { years. } \\
\text { Low Performance. } \\
\leq 200 \mathrm{~m}: n=23(26.7 \%) .57 .6 \pm \\
20.0 \text { years. }\end{array}$ & $\begin{array}{l}\text { Descompensated } \\
\text { HFpEF and HFrEF } \\
\text { LVEF }<40 \% \text { (HFrEF) } \\
\text { LVEF } \geq 40 \% \\
\text { (HFpEF) }\end{array}$ \\
\hline $\begin{array}{l}\text { Zotter-Tufaro et al. } \\
\text { [58], } 2015 .\end{array}$ & Austria & Not Reported & Prospective & $\begin{array}{l}\text { High Performance. } \\
>300 \mathrm{~m}: n=72(31.95 \%) .67 .8 \pm \\
9.1 \text { years. } \\
\text { Low Performance. } \\
\leq 300 \mathrm{m:} n=70 \text { (28.6\%). } 73.1 \pm \\
7.4 \text { years. }\end{array}$ & $\begin{array}{l}\text { HFpEF } \\
\text { LVEF } \geq 50 \%\end{array}$ \\
\hline Boxer et al. [59], 2010. & USA & $\begin{array}{l}\text { University of Connecticut } \\
\text { Health Centre }\end{array}$ & Prospective & $\begin{array}{l}\text { All: } n=60 \text { (71.66\%). } 78 \pm 12.0 \\
\text { years. } \\
\text { High Performance. } \\
>300 \text { m. NS. } \\
\text { Low Performance. } \\
\leq 300 \text { m. NS. }\end{array}$ & $\begin{array}{l}\text { HFrEF } \\
\text { LVEF } \leq 40 \%\end{array}$ \\
\hline Ingle et al. [60], 2014 & $\begin{array}{l}\text { United } \\
\text { Kingdom }\end{array}$ & Not Reported & Prospective & $\begin{array}{l}\text { All: } n=600(75 \%) .77 .8(71.5- \\
83.6) \text { years. } \\
\text { High Performance. } \\
>365 \mathrm{~m} . n=\mathrm{NS} \text {. } \\
\text { Middle Performance. } \\
271-365 \mathrm{~m} . n=\mathrm{NS} \text {. } \\
\text { Low Performance. } \\
61-270 \mathrm{~m} . n=\text { NS. } \\
\text { Very Low Performance. } \\
<60 \mathrm{~m} . n=\text { NS. }\end{array}$ & $\begin{array}{l}\text { HFrEF } \\
\text { LVEF }<45 \%\end{array}$ \\
\hline Guazzi et al. [61], 2009. & Italy & $\begin{array}{l}\text { Clinical Care Setting } \\
\text { (Secondary Care) }\end{array}$ & Prospective & $\begin{array}{l}\text { All: } n=253(78.66 \%) .61 .9 \pm 10.1 \\
\text { years. }\end{array}$ & $\begin{array}{l}\text { HFpEF and HFrEF } \\
\text { LVEF }<50 \%\end{array}$ \\
\hline
\end{tabular}


Table 1 Characteristics of included studies (Continued)

\begin{tabular}{|c|c|c|c|c|c|}
\hline $\begin{array}{l}\text { Study (first author and } \\
\text { year) }\end{array}$ & Region & Setting & Design & $\begin{array}{l}\text { Study Characteristics: Groups, } \\
\text { Sample Size (\%Male), Age }\end{array}$ & $\begin{array}{l}\text { Heart Failure } \\
\text { Diagnosis }\end{array}$ \\
\hline & & & & $\begin{array}{l}\text { High Performance. } \\
>300 \mathrm{~m} . n=175 . \mathrm{NS} \text {. } \\
\text { Low Performance. } \\
\leq 300 \mathrm{~m} . n=78 \text {. NS }\end{array}$ & $\begin{array}{l}\text { (HFrEF) } \\
\text { LVEF } \geq 50 \% \\
\text { (HFpEF) }\end{array}$ \\
\hline $\begin{array}{l}\text { McCabe et al. [62], } \\
2017 .\end{array}$ & USA & An University Hospital & Prospective & $\begin{array}{l}\text { All: } n=71 \text { ( } 57.7 \%) .52 .6 \pm 12.3 \\
\text { years. } \\
\text { High Performance. } \\
>300 \mathrm{~m} . \text { NS. } \\
\text { Low Performance. } \\
\leq 300 \mathrm{~m} . \mathrm{NS} \text {. }\end{array}$ & $\begin{array}{l}\text { HFpEF and HFrEF } \\
\mathrm{LVEF}=24.4 \pm 13.5\end{array}$ \\
\hline Vegh et al. [63], 2014. & USA & $\begin{array}{l}\text { Clinical Care Setting } \\
\text { (Secondary Care) }\end{array}$ & Prospective & $\begin{array}{l}\text { All: } n=164 \text { (77\%). } 67.3 \pm 12.9 \\
\text { years. } \\
\text { High Performance. } \\
\geq 350 \mathrm{~m} \text {. NS. } \\
\text { Middle Performance. } \\
280-350 \mathrm{~m} \text {. NS. } \\
\text { Low Performance. } \\
<280 \mathrm{~m} \text {. NS. }\end{array}$ & $\begin{array}{l}\mathrm{HFrEF} \\
\mathrm{LVEF}=25 \% \pm 7 \%\end{array}$ \\
\hline Roul et al. [64], 1998. & France & Not Reported & Prospective & $\begin{array}{l}\text { All: } n=121 \text { ( } 81.8 \%) .59 \pm 11 \\
\text { years. } \\
\text { High Performance. } \\
>300 \mathrm{~m} . \text { NS. } \\
\text { Low Performance. } \\
\leq 300 \mathrm{~m} . \mathrm{NS} \text {. }\end{array}$ & $\begin{array}{l}\text { HFrEF } \\
\text { LVEF }=29.6 \% \pm 13 \%\end{array}$ \\
\hline $\begin{array}{l}\text { Frankenstein et al. [65], } \\
2008 .\end{array}$ & Germany & $\begin{array}{l}\text { Specialised HF clinic at the } \\
\text { University of Heidelberg }\end{array}$ & Prospective & $\begin{array}{l}\text { All: } n=1035(80.2 \%) 54.9 \pm 11.5 \\
\text { years. } \\
\text { Mean 6MWT: } 459 \mathrm{~m} \pm 113 \mathrm{~m}\end{array}$ & $\begin{array}{l}\text { HFrEF } \\
\text { LVEF } \leq 40 \%\end{array}$ \\
\hline $\begin{array}{l}\text { Mene-Afejuku et al. } \\
\text { [66], 2017. }\end{array}$ & Nigeria & Not Reported & Prospective & $\begin{array}{l}\text { All: } n=100 \text { (NS). } 64.02 \pm 12.88 \\
\text { years. } \\
\text { High Performance. } \\
314.66 \mathrm{~m} \pm 48.17 \mathrm{~m} . n=59 \text { (NS). } \\
66.32 \pm 12.29 \text { years. } \\
\text { Low Performance. } \\
260.59 \mathrm{~m} \pm 66.65 \mathrm{~m} . \\
61.71 \pm 13.46 \text { years. }\end{array}$ & $\begin{array}{l}\text { HHF (HFrEF and } \\
\text { HFpEF) } \\
\text { LVEF } \leq 40 \% \text { (HFrEF) } \\
\text { LVEF }>40 \% \\
\text { (HFpEF) }\end{array}$ \\
\hline Ingle et al. [67], 2007 & $\begin{array}{l}\text { United } \\
\text { Kingdom }\end{array}$ & Not Reported & Prospective & $\begin{array}{l}\text { All: } n=1592(60 \%) .74 \text { (67-80) } \\
\text { years. } \\
\text { High Performance. } \\
\geq 421 \mathrm{~m} \text {. NS. } \\
\text { Middle Performance. } \\
346-420 \mathrm{~m} \text {. NS. } \\
\text { Low Performance. } \\
241-345 \mathrm{~m} \text {. NS. } \\
\text { Very Low Performance. } \\
\geq 240 \mathrm{~m} \text {. NS. }\end{array}$ & $\begin{array}{l}\text { HFrEF } \\
\text { LVEF } \leq 45 \%\end{array}$ \\
\hline $\begin{array}{l}\text { Rostagno et al. [68], } \\
2003 \text {. }\end{array}$ & Italy & $\begin{array}{l}\text { Clinical Care Setting } \\
\text { (Secondary Care) }\end{array}$ & Prospective & $\begin{array}{l}\text { All: } n=214 \text { (93\%). } 53.7 \text { (29-70) } \\
\text { years. } \\
\text { High Performance. } \\
\geq 450 \text { m. NS. } \\
\text { Middle Performance. } \\
300-450 \mathrm{~m} \text {. NS. } \\
\text { Low Performance. } \\
<300 \text { m. NS. }\end{array}$ & $\begin{array}{l}\text { Congestive HFpEF } \\
\text { and HFrEF } \\
\text { LVEF }<50 \% \text { (HFrEF) } \\
\text { LVEF } \geq 50 \% \\
\text { (HFpEF) }\end{array}$ \\
\hline $\begin{array}{l}\text { Cahalin et al. [69], } \\
1996 .\end{array}$ & USA & $\begin{array}{l}\text { Clinical Care Setting } \\
\text { (Secondary Care) }\end{array}$ & Prospective & $\begin{array}{l}\text { All: } n=45 \text { ( } 89 \%) .49 \pm 8 \text { years. } \\
\text { High Performance. } \\
\geq 300 \text { m. NS. } \\
\text { Low Performance. } \\
<300 \text { m. NS. }\end{array}$ & $\begin{array}{l}\text { HFrEF } \\
\text { LVEF }=20 \pm 6\end{array}$ \\
\hline $\begin{array}{l}\text { Frankenstein et al. [70], } \\
2008 .\end{array}$ & Germany & $\begin{array}{l}\text { Specialised HF clinic at the } \\
\text { University of Heidelberg }\end{array}$ & Prospective & $\begin{array}{l}\text { All: } n=1069(80.6 \%) 55.2 \pm 11.7 \\
\text { years. } \\
\text { Mean 6MWT: } 456 \mathrm{~m} \pm 114 \mathrm{~m}\end{array}$ & $\begin{array}{l}\text { HFrEF } \\
\text { LVEF }=29 \% \pm 10 \%\end{array}$ \\
\hline Rubim et al. [71], 2006. & Brazil & Clinical Care Setting & Prospective & All: $n=176$ (67\%). $58.32 \pm 12.7$ & HFpEF and HFrEF \\
\hline
\end{tabular}


Table 1 Characteristics of included studies (Continued)

\begin{tabular}{|c|c|c|c|c|c|}
\hline $\begin{array}{l}\text { Study (first author and } \\
\text { year) }\end{array}$ & Region & Setting & Design & $\begin{array}{l}\text { Study Characteristics: Groups, } \\
\text { Sample Size (\%Male), Age }\end{array}$ & $\begin{array}{l}\text { Heart Failure } \\
\text { Diagnosis }\end{array}$ \\
\hline & & (Secondary Care) & & $\begin{array}{l}\text { years. } \\
\text { Mean 6MWT: } 521.11 \mathrm{~m} \pm 76.1 \mathrm{~m} \text {. } \\
\text { High Performance. } \\
\geq 520 \mathrm{~m} \text {. NS. } \\
\text { Low Performance. } \\
<520 \mathrm{~m} \text {. NS. }\end{array}$ & $\begin{array}{l}\mathrm{LVEF}=34.91 \% \\
\pm 12.4 \%\end{array}$ \\
\hline $\begin{array}{l}\text { Kanagala et al. [72], } \\
2019 .\end{array}$ & United Kingdom & $\begin{array}{l}\text { Clinical Care Setting (Tertiary } \\
\text { Care Hospital) }\end{array}$ & Prospective & $\begin{array}{l}\text { All: } n=140(49 \%) .73 \pm 9.0 \text { years. } \\
\text { Mean 6MWT: } 180 \text { m ( } 120 \text { m- } \\
250 \mathrm{~m})\end{array}$ & $\begin{array}{l}\text { HFpEF and HFrEF } \\
\text { LVEF }>50 \%\end{array}$ \\
\hline Zugck et al. [73], 2001. & Germany & $\begin{array}{l}\text { Medical Clinic of the } \\
\text { University of } \\
\text { Heidelberg }\end{array}$ & Prospective & $\begin{array}{l}\text { All: } n=208(82 \%) .54 \pm 10 \text { years. } \\
\text { Mean 6MWT: } 455 \mathrm{~m} \pm \\
107 \mathrm{~m}(170 \mathrm{~m}-692 \mathrm{~m})\end{array}$ & $\begin{array}{l}\text { HFrEF } \\
\text { LVEF } \leq 40 \%\end{array}$ \\
\hline Cahalin et al. [74], 2013. & Italy & $\begin{array}{l}\text { Clinical Care Setting } \\
\text { (Secondary Care) }\end{array}$ & Prospective & $\begin{array}{l}\text { All: } n=258 \text { (NS). } 63 \pm 8.7 \text { years. } \\
\text { High Performance. } \\
>300 \mathrm{~m} \text {. NS. } \\
\text { Low Performance. } \\
\leq 300 \mathrm{~m} \text {. NS. }\end{array}$ & $\begin{array}{l}\text { HFpEF and HFrEF } \\
\text { LVEF }<50 \% \text { (HFrEF) } \\
\text { LVEF } \geq 50 \% \\
\text { (HFpEF) }\end{array}$ \\
\hline Reibis et al. [75], 2010. & Germany & $\begin{array}{l}\text { Clinical Care Setting } \\
\text { (Secondary Care) }\end{array}$ & Prospective & $\begin{array}{l}\text { All: } n=1346(73 \%) .64 \pm 10 \\
\text { years. } \\
\text { Mean } 6 \text { MWT: } 350.1 \mathrm{~m} \pm 148.6 \mathrm{~m}\end{array}$ & $\begin{array}{l}\text { HFrEF } \\
\text { LVEF }<45 \%\end{array}$ \\
\hline Castel et al. [76], 2009. & Spain & Not Reported & Retrospective & $\begin{array}{l}\text { All: } n=155(82 \%) .68 .6 \pm 7.8 \\
\text { years. } \\
\text { High Performance. } \\
>400 \mathrm{~m} \text {. NS. } \\
\text { Middle Performance. } \\
310-400 \mathrm{~m} \text {. NS. } \\
\text { Low Performance. } \\
\text { 225-310 m. NS. } \\
\text { Very Low Performance. } \\
<225 \mathrm{~m} \text {. NS. }\end{array}$ & $\begin{array}{l}\text { HFrEF } \\
\text { LVEF } \leq 45 \%\end{array}$ \\
\hline $\begin{array}{l}\text { Kamiya et al. [77], } \\
2017 .\end{array}$ & Japan & $\begin{array}{l}\text { Clinical Care Setting } \\
\text { (Secondary Care Centre) }\end{array}$ & Retrospective & $\begin{array}{l}\text { All: } n=1474 \text { (68\%). } 72.2 \pm 7.1 \\
\text { years. } \\
\text { High Performance. } \\
\geq 446 \mathrm{m:} n=485 \text { (84\%). } 68.5 \pm \\
5.6 \text { years. } \\
\text { Middle Performance. } \\
342-445 \mathrm{~m}: n=497 \text { (69\%). } 71.5 \pm \\
6.3 \text { years. } \\
\text { Low Performance. } \\
\leq 341 \mathrm{~m}: n=492 \text { (52\%). } 76.5 \pm \\
7.0 \text { years. }\end{array}$ & $\begin{array}{l}\text { HFpEF and HFrEF } \\
\text { LVEF }=52.7 \pm 15.4\end{array}$ \\
\hline \multicolumn{6}{|c|}{ Short Physical Performance Battery (SPPB) } \\
\hline García et al. [78], 2019. & Spain & $\begin{array}{l}\text { Clinical Care Setting } \\
\text { (Secondary Care) }\end{array}$ & Prospective & $\begin{array}{l}\text { High Performance. } \\
\text { SPPB }>7: n=37(54.1 \%) .83 \pm \\
5.7 \text { years. } \\
\text { Low Performance. } \\
\text { SPPB } \leq 7: n=49 \text { (30.6\%). } 86 \pm 6.7 \\
\text { years. }\end{array}$ & Acute HF \\
\hline $\begin{array}{l}\text { Hornsby et al. [79], } \\
2019 .\end{array}$ & USA & University of Michigan & Prospective & $\begin{array}{l}\text { High Performance. } \\
\text { SPPB } \geq 10 \text { points: } n=22(55 \%) \text {. } \\
64 \pm 13.0 \text { years. } \\
\text { Middle Performance. } \\
\text { SPPB }=7-9 \text { points: } n=53(42 \%) \text {. } \\
67 \pm 12.0 \text { years. } \\
\text { Low Performance. } \\
\text { SPPB } 6 \text { points: } n=39(36 \%) . \\
72 \pm 13.0 \text { years. }\end{array}$ & $\begin{array}{l}\text { HFpEF HF } \\
\text { LVEF } \geq 50 \%\end{array}$ \\
\hline $\begin{array}{l}\text { Chiarantini et al. [80], } \\
2010 .\end{array}$ & Italy & $\begin{array}{l}\text { Clinical Care Setting } \\
\text { (Secondary Care) }\end{array}$ & Prospective & $\begin{array}{l}\text { All: } n=157 \text { (50.3\%). } 80 \pm 0.5 \\
\text { years. } \\
\text { High Performance. } \\
\text { SPPB }=9-12: n=32 . \text { NS. } \\
\text { Middle Performance. }\end{array}$ & $\begin{array}{l}\text { Descompensated } \\
\text { HFrEF and HFpEF } \\
\text { LVEF }<45 \% \text { (HFrEF) } \\
\text { LVEF } \geq 45 \% \\
\text { (HFpEF) }\end{array}$ \\
\hline
\end{tabular}


Table 1 Characteristics of included studies (Continued)

\begin{tabular}{|c|c|c|c|c|c|}
\hline $\begin{array}{l}\text { Study (first author and } \\
\text { year) }\end{array}$ & Region & Setting & Design & $\begin{array}{l}\text { Study Characteristics: Groups, } \\
\text { Sample Size (\%Male), Age }\end{array}$ & $\begin{array}{l}\text { Heart Failure } \\
\text { Diagnosis }\end{array}$ \\
\hline & & & & $\begin{array}{l}\text { SPPB }=5-8: n=45 . \text { NS. } \\
\text { Low Performance. } \\
\text { SPPB }=1-4: n=33 . \text { NS. } \\
\text { Very Low Performance. } \\
\text { SPPB }=0: n=47 . \text { NS. }\end{array}$ & \\
\hline $\begin{array}{l}\text { Zaharias et al. [81], } \\
2014 .\end{array}$ & USA & $\begin{array}{l}\text { Clinical Care Setting } \\
\text { (Secondary Care) }\end{array}$ & Prospective & $\begin{array}{l}\text { All: } n=32 \text { (78.1\%). } 58.2 \pm 13.6 \\
\text { years. } \\
\text { High Performance. } \\
\text { SPPB }=10-12: n=7 . \text { NS. } \\
\text { Middle Performance. } \\
\text { SPPB }=7-9: n=8 . \text { NS. } \\
\text { Low Performance. } \\
\text { SPPB }=4-6: n=12 . \text { NS. } \\
\text { Very Low Performance. } \\
\text { SPPB }=0-3: n=4 . \text { NS. }\end{array}$ & $\begin{array}{l}\text { HFrEF and HFpEF } \\
\text { LVEF }<40 \% \text { (HFrEF) } \\
\text { LVEF } \geq 40 \% \\
\text { (HFpEF) }\end{array}$ \\
\hline
\end{tabular}

\section{Gait Speed (GS)}

Lo et al. [82], 2015.

USA

Pulignano et al. [83], 2016.

Italy

Chaudhry et al. [84], USA 2013

Tanaka et al. [85], 2018. Japan

Tanaka et al. [86], 2019. Japan

Rodríguez-Pascual Spain et al. [87], 2017.

Vidán et al. [88], 2016. Spain
Community Based Population

Clinical Care Setting (7 Secondary Care Centres)

Not Reported

Kitasato University Hospital

Kitasato University Hospital

Clinical Care Setting (6 Secondary Care Centres)

Clinical Care Setting (Secondary Care Centre)
Prospective High Performance.

GS $\geq 0.8 \mathrm{~m} / \mathrm{s}: n=553$ (59\%).

$73 \pm 5.0$ years.

Low Performance.

GS $<0.8 \mathrm{~m} / \mathrm{s}: n=566$ (39\%).

$76 \pm 6.0$ years.

Prospective High Performance.

GS $\geq 1.0 \mathrm{~m} / \mathrm{s}: n=88$ (64.8\%).

$76.4 \pm 4.8$ years.

Middle Performance.

$\mathrm{GS}=0.66-0.99 \mathrm{~m} / \mathrm{s}: n=128$

(60.9\%). $77.1 \pm 4.7$ years

Low Performance.

GS $\leq 0.65 \mathrm{~m} / \mathrm{s}: n=115$ (48.7\%).

$80.2 \pm 5.6$ years.

Prospective

All: $n=758$ (49.5\%). $79.7 \pm 6.2$ years.

High Performance.

GS $>0.8 \mathrm{~m} / \mathrm{s}: n=441$. NS.

Low Performance.

GS $\leq 0.8 \mathrm{~m} / \mathrm{s}: n=317$. NS.

Retrospective All: $n=603$ (62.7\%). $74.9 \pm 6.2$ years.

High Performance.

GS $>1.14 \mathrm{~m} / \mathrm{s}: n=154$. NS.

Middle Performance.

$\mathrm{GS}=1.0-1.14 \mathrm{~m} / \mathrm{s} . n=149$. NS.

Low Performance.

$\mathrm{GS}=0.82-0.99 \mathrm{~m} / \mathrm{s} . n=150$. NS

Very Low Performance.

GS $<0.82 \mathrm{~m} / \mathrm{s}: n=150$. NS.

Retrospective High Performance.

GS $\geq 0.8 \mathrm{~m} / \mathrm{s}: n=194$ (72.7\%).

$73.1 \pm 6.7$ years.

Low Performance.

GS < $0.8 \mathrm{~m} / \mathrm{s}: n=194$ (44.8\%).

$76.5 \pm 8.4$ years.

Prospective

High Performance.

$\mathrm{GS} \geq 0.65 \mathrm{~m} / \mathrm{s}: n=211$ (47.9\%).

$84.4 \pm 9.4$ years.

Low Performance.

GS < $0.65 \mathrm{~m} / \mathrm{s}: n=286$ (32.5\%).

$85.7 \pm 5.1$ years.

Prospective

All: $n=416$ (50.5\%). $80.0 \pm 6.1$

years.

High Performance.
HFpEF and HFrEF

LVEF $<45 \%$ (HFrEF)

LVEF $\geq 45 \%$

(HFpEF)

HFpEF and HFrEF LVEF $<45 \%$ (HFrEF)

LVEF $\geq 45 \%$

(HFpEF)

HFpEF and HFrEF

LVEF $<45 \%$ (HFrEF)

LVEF $\geq 45 \%$

(HFpEF)

Acute HFpEF and

HFrEF

LVEF $<40 \%$ (HFrEF)

LVEF $\geq 40 \%$

(HFpEF)

Acute HFpEF and HFrEF

LVEF $<40 \%$ (HFrEF)

LVEF $\geq 40 \%$

(HFpEF)

HFpEF and HFrEF

LVEF $\leq 45 \%$ (HFrEF)

LVEF > $45 \%$

(HFpEF)

HFpEF and HFrEF

LVEF $<50 \%$ (HFrEF)

$\mathrm{LVEF} \geq 45 \%$ 
Table 1 Characteristics of included studies (Continued)

\begin{tabular}{|c|c|c|c|c|c|}
\hline $\begin{array}{l}\text { Study (first author and } \\
\text { year) }\end{array}$ & Region & Setting & Design & $\begin{array}{l}\text { Study Characteristics: Groups, } \\
\text { Sample Size (\%Male), Age }\end{array}$ & $\begin{array}{l}\text { Heart Failure } \\
\text { Diagnosis }\end{array}$ \\
\hline & & & & $\begin{array}{l}\mathrm{GS} \geq 0.65 \mathrm{~m} / \mathrm{s} . \mathrm{NS} \\
\text { Low Performance. } \\
\mathrm{GS}<0.65 \mathrm{~m} / \mathrm{s} . \mathrm{NS} \text {. }\end{array}$ & $\begin{array}{l}\text { (HFpEF) } \\
\text { LVEF }=43.4 \% \pm \\
14.7 \%\end{array}$ \\
\hline $\begin{array}{l}\text { Kamiya et al. [77], } \\
2017 .\end{array}$ & Japan & $\begin{array}{l}\text { Clinical Care Setting } \\
\text { (Secondary Care Centre) }\end{array}$ & Retrospective & $\begin{array}{l}\text { All: } n=1474(68 \%) .72 .2 \pm 7.1 \\
\text { years. } \\
\text { High Performance. } \\
\text { GS } \geq 1.17 \mathrm{~m} / \mathrm{s}: n=489(82 \%) \\
68.7 \pm 5.5 \text { years. } \\
\text { Middle Performance. } \\
\text { GS }=0.95-1.160 \mathrm{~m} / \mathrm{s}: n=489 \\
(67 \%) .71 .8 \pm 6.6 \text { years. } \\
\text { Low Performance. } \\
\text { GS } \geq 0.94 \mathrm{~m} / \mathrm{s}: n=496(55 \%) \\
76.1 \pm 7.2 \text { years. }\end{array}$ & $\begin{array}{l}\text { HFpEF and HFrEF } \\
\text { LVEF }=52.7 \pm 15.4\end{array}$ \\
\hline
\end{tabular}

$m$ Meters. HF Heart Failure. LVEF Left Ventricular Ejection Fraction. NS Not Specified. HFrEF Patients with Heart Failure with Reduced Ejection Fraction (Systolic Heart Failure). HFPEF Patients with Heart Failure with Preserved Ejection Fraction (Diastolic Heart Failure). HHF Hypertensive Heart Failure. SPPB Short Physical Performance Battery. GS Gait Speed

decreased the meters $(\mathrm{m})$ they walked in the 6MWT during follow-up showed larger risk of All-Cause of Mortality $[\mathrm{HR}=1.22$ 95\%CI $(1.10-1.36), p<0.001]$, although there was no lower risk of All-Cause of Mortality between patients with HFrEF, patients with HFpEF and patients with acute HF who increased the meters they walked in the 6MWT during follow-up (Additional file 5). Patients with HFrEF and HFpEF who showed a poor physical functional performance in the 6MWT also reported a larger risk of HF Mortality [HR = 2.39 95\%CI $(2.21-2.59), p<0.001]$ than those patients who showed a good physical functional performance (Fig. 2b). Patients with HFrEF who showed a poor physical functional performance in the 6MWT also reported a larger risk of the combined endpoint of Hospitalisation and Mortality for any cause [HR $=1.8095 \% \mathrm{CI}(1.45-$ $2.23), p<0.001]$ or $[\mathrm{OR}=2.0795 \% \mathrm{CI}(1.41-3.02), p<$ 0.001 ] than those patients who showed a good physical functional performance (Fig. 2c and Fig. 2d, respectively). Furthermore, patients with HFrEF, HFpEF and acute HF who showed a poor physical functional performance in the 6MWT reported a larger risk of HF Hospitalisation $[\mathrm{HR}=1.6895 \% \mathrm{CI}(1.20-2.33), p=0.002$ ] than those patients who showed a good physical functional performance (Additional file 5). On the other hand, patients with HFrEF, HFpEF and acute HF who showed a slower gait speed reported a larger risk of AllCause of Mortality [HR $=1.49$ 95\%CI (1.24-1.79), $p<$ 0.001] than those patients who showed a faster gait speed (Fig. 3), above all, when gait speed was slower than $0.65 \mathrm{~m} / \mathrm{s} \quad[\mathrm{HR}=1.5995 \% \mathrm{CI}(1.10-2.30), p=0.01]$ (Additional file 5). Moreover, patients with HFrEF, HFpEF and acute HF who increased their gait speed during follow-up showed a lower risk of All-Cause of Mortality [HR $=0.85$ 95\%CI (0.81-0.91) (Additional file 5). Patients with HFrEF and HFpEF who showed a slower gait speed $(<0.80 \mathrm{~m} / \mathrm{s})$ also reported a larger risk of All-Cause of Hospitalisation [HR $=1.32$ 95\%CI (1.101.57), $p=0.002$ ] than patients with a faster gait speed (> $0.80 \mathrm{~m} / \mathrm{s}$ ) (Additional file 5).

\section{Sensitivity analyses}

The risk of All-Cause of Mortality in the 6MWT was larger when only patients with HFrEF and poor physical functional performance were assessed $[\mathrm{HR}=2.4695 \% \mathrm{CI}$ (1.94-3.12), $p<0.001$ ] (Additional file 6). However, the risk of HF Mortality [HR $=2.39$ 95\%CI $(2.21-2.58), p<$ 0.001 ] as well as the risk of All-Cause of Mortality in the 6MWT per increased units did not change when only patients with HFrEF were assessed (Additional file 6).

\section{Descriptive quantitative analysis}

\section{Physical functional performance and mortality}

A score between 1 and 4 points on the SPPB was associated with a larger risk of All-Cause of Mortality ( $\mathrm{HR}=$ 4.78 95\% CI [1.63-14.02, $p<0.05])$ in patients with HFrEF and HFpEF [80], while a score below 7 points on the SPPB was not associated with a larger risk of AllCause of Mortality in patients with acute HF [78].

\section{Physical functional performance and the combined endpoint of hospitalisation and mortality}

A score below 7 points on the SPPB was associated with a larger risk of the combined endpoint of hospitalisation and mortality for any cause $(\mathrm{OR}=3.695 \% \mathrm{CI}[1.0-12.9$, $p<0.05])$ in patients with acute HF [78]. However, per each 1-unit improved in SPPB the risk of the combined endpoint of hospitalisation and mortality for any cause could be reduced OR $=0.8195 \% \mathrm{CI}[0.69-0.94, p<0.05]$ in patients with HFpEF [79]. Patients with HFrEF and HFpEF with a gait speed slower than $0.8 \mathrm{~m} / \mathrm{s}$ also showed a larger risk of the combined endpoint of 
Table 2 Outcomes, Results, Risk of Bias of Included Studies and Level of Evidence per Outcome according to GRADE Criteria

\begin{tabular}{|c|c|c|c|c|c|c|}
\hline $\begin{array}{l}\text { Study (first author and } \\
\text { year) }\end{array}$ & $\begin{array}{l}\text { Functional } \\
\text { Test }\end{array}$ & Follow-Up & Outcomes & Main Results & $\begin{array}{l}\text { Risk of } \\
\text { Bias }\end{array}$ & $\begin{array}{l}\text { Level of } \\
\text { Evidence } \\
\text { (GRADE) }\end{array}$ \\
\hline \multirow[t]{4}{*}{ Brenyo et al. [45], 2012.} & $6 \mathrm{MWT}$ & 4 years & $\begin{array}{l}\text { Incident HF and Mortality } \\
\leq 350 \mathrm{~m} \mathrm{VS}>350 \mathrm{~m}\end{array}$ & $\begin{array}{l}\mathrm{HR}=1.7395 \% \mathrm{Cl}[1.29- \\
2.33]^{* * *}\end{array}$ & Low & $\begin{array}{l}\text { Not } \\
\text { Reported }\end{array}$ \\
\hline & & & $\begin{array}{l}\text { Incident HF and Mortality } \\
\text { Per } 100-\mathrm{m} \text { decreased }\end{array}$ & $\begin{array}{l}\mathrm{HR}=1.2595 \% \mathrm{Cl}[1.09- \\
1.44]^{* * *}\end{array}$ & & \\
\hline & & & $\begin{array}{l}\text { All-Cause Mortality } \\
\leq 350 \mathrm{~m} \text { VS }>350 \mathrm{~m}\end{array}$ & $\begin{array}{l}\mathrm{HR}=2.4095 \% \mathrm{Cl}[1.42- \\
4.08]^{* * *}\end{array}$ & & Moderate \\
\hline & & & $\begin{array}{l}\text { All-Cause Mortality } \\
\text { Per 100-m decreased }\end{array}$ & $\begin{array}{l}\mathrm{HR}=1.3295 \% \mathrm{Cl}[1.05- \\
1.66]^{* *}\end{array}$ & & \\
\hline \multirow[t]{6}{*}{ Ferreira et al. [46], 2019.} & $6 \mathrm{MWT}$ & $\begin{array}{l}21 \text { months (9-26 } \\
\text { months) }\end{array}$ & $\begin{array}{l}\text { Hospitalisation and } \\
\text { Mortality } \\
241-360 m \text { VS }>360 m\end{array}$ & $\begin{array}{l}\mathrm{HR}=1.4495 \% \mathrm{Cl}[1.14- \\
1.80]^{* *}\end{array}$ & Moderate & Low \\
\hline & & & $\begin{array}{l}\text { Hospitalisation and } \\
\text { Mortality } \\
\leq 240 m \text { VS }>360 m\end{array}$ & $\begin{array}{l}\mathrm{HR}=1.7395 \% \mathrm{Cl}[1.38- \\
2.18]^{* * *}\end{array}$ & & \\
\hline & & & $\begin{array}{l}\text { Hospitalisation and } \\
\text { Mortality } \\
\text { Per each } 50 \mathrm{~m} \text { decreased }\end{array}$ & $\begin{array}{l}\mathrm{HR}=1.0895 \% \mathrm{Cl}[1.04- \\
1.11]^{* * *}\end{array}$ & & \\
\hline & & & $\begin{array}{l}\text { All-Cause Mortality } \\
241-360 m \text { VS }>360 m\end{array}$ & $\begin{array}{l}\mathrm{HR}=1.4995 \% \mathrm{Cl}[1.08- \\
2.06]^{* *}\end{array}$ & & Moderate \\
\hline & & & $\begin{array}{l}\text { All-Cause Mortality } \\
\leq 240 \mathrm{~m} \text { VS }>360 \mathrm{~m}\end{array}$ & $\begin{array}{l}\mathrm{HR}=2.4195 \% \mathrm{Cl}[1.76- \\
3.29]^{* * *}\end{array}$ & & \\
\hline & & & $\begin{array}{l}\text { All-Cause Mortality } \\
\text { Per each } 50 \text { m decreased }\end{array}$ & $\begin{array}{l}\mathrm{HR}=1.1495 \% \mathrm{Cl}[1.09- \\
1.18]^{* * *}\end{array}$ & & \\
\hline \multirow[t]{4}{*}{$\begin{array}{l}\text { Wegrzynowska-Teodorczyk } \\
\text { et al. [47], } 2013 .\end{array}$} & $6 \mathrm{MWT}$ & 1 year & $\begin{array}{l}\text { HF Mortality } \\
\leq 468 \mathrm{~m} \mathrm{VS}>468 \mathrm{~m}\end{array}$ & $\begin{array}{l}\mathrm{HR}=3.2295 \% \mathrm{Cl}[1.17- \\
8.86]^{* *}\end{array}$ & Low & Moderate \\
\hline & & & $\begin{array}{l}\text { Hospitalisation and } \\
\text { Mortality } \\
\leq 468 m \text { VS }>468 m\end{array}$ & $\begin{array}{l}\mathrm{HR}=2.7795 \% \mathrm{Cl}[1.30- \\
5.88]^{* *}\end{array}$ & & Low \\
\hline & & 3 years & $\begin{array}{l}\text { HF Mortality } \\
\leq 468 \mathrm{~m} \mathrm{VS}>468 \mathrm{~m}\end{array}$ & $\begin{array}{l}\mathrm{HR}=2.1895 \% \mathrm{Cl}[1.18- \\
4.03]^{* *}\end{array}$ & & Moderate \\
\hline & & & $\begin{array}{l}\text { Hospitalisation and } \\
\text { Mortality } \\
\leq 468 m \text { VS }>468 m\end{array}$ & $\begin{array}{l}\mathrm{HR}=1.7195 \% \mathrm{Cl}[1.08- \\
2.72]^{* *}\end{array}$ & & Low \\
\hline \multirow[t]{9}{*}{ Bittner et al. [48], 1993.} & $6 \mathrm{MWT}$ & 1 year ( $242 \pm 82$ days) & $\begin{array}{l}\text { All-Cause Mortality } \\
\text { Per each } 120 \text { m decreased }\end{array}$ & $\begin{array}{l}\mathrm{OR}=1.5095 \% \mathrm{Cl}[1.11- \\
2.03]^{* *}\end{array}$ & Low & Moderate \\
\hline & & & $\begin{array}{l}\text { HF Hospitalisation } \\
\text { Per each } 120 \text { m decreased }\end{array}$ & $\begin{array}{l}\mathrm{OR}=2.6095 \% \mathrm{Cl}[1.78- \\
3.80]^{* * *}\end{array}$ & & Low \\
\hline & & & $\begin{array}{l}\text { Hospitalisation and } \\
\text { Mortality } \\
\text { Per each } 120 \mathrm{~m} \text { decreased }\end{array}$ & $\begin{array}{l}\mathrm{OR}=1.7795 \% \mathrm{Cl}[1.38- \\
2.26]^{* * *}\end{array}$ & & Low \\
\hline & & & $\begin{array}{l}\text { All-Cause Mortality } \\
<300 \mathrm{~m} \mathrm{VS} \geq 450 \mathrm{~m}\end{array}$ & $\begin{array}{l}\mathrm{OR}=3.795 \% \mathrm{Cl}[1.44- \\
9.55]^{* *}\end{array}$ & & Moderate \\
\hline & & & $\begin{array}{l}\text { All-Cause Mortality } \\
300-375 \mathrm{~m} \text { VS } \geq 450 \mathrm{~m}\end{array}$ & $\begin{array}{l}\mathrm{OR}=2.7895 \% \mathrm{Cl}[1.09- \\
7.11]^{* *}\end{array}$ & & \\
\hline & & & $\begin{array}{l}\text { All-Cause Mortality } \\
375-450 \mathrm{~m} \text { VS } \geq 450 \mathrm{~m}\end{array}$ & $\begin{array}{l}\mathrm{OR}=1.4295 \% \mathrm{Cl}[0.50- \\
4.06]^{*}\end{array}$ & & \\
\hline & & & $\begin{array}{l}\text { All-Cause Hospitalisation } \\
<300 \mathrm{~m} \text { VS } \geq 450 \mathrm{~m}\end{array}$ & $\begin{array}{l}\mathrm{OR}=14.0295 \% \mathrm{Cl}[4.90- \\
40.14]^{* * *}\end{array}$ & & Low \\
\hline & & & $\begin{array}{l}\text { All-Cause Hospitalisation } \\
300-375 \mathrm{~m} \text { VS } \geq 450 \mathrm{~m}\end{array}$ & $\begin{array}{l}\mathrm{OR}=6.2195 \% \mathrm{Cl}[2.14- \\
18.08]^{* * *}\end{array}$ & & \\
\hline & & & $\begin{array}{l}\text { All-Cause Hospitalisation } \\
375-450 \mathrm{~m} \text { VS } \geq 450 \mathrm{~m}\end{array}$ & $\begin{array}{l}\mathrm{OR}=1.9095 \% \mathrm{Cl}[0.56- \\
6.42]^{*}\end{array}$ & & \\
\hline Arslan et al. [49], 2007. & $6 \mathrm{MWT}$ & 2 years $(18 \pm 6$ & HF Mortality & $\mathrm{HR}=2.3895 \% \mathrm{Cl}[2.02-$ & Moderate & Moderate \\
\hline
\end{tabular}


Table 2 Outcomes, Results, Risk of Bias of Included Studies and Level of Evidence per Outcome according to GRADE Criteria (Continued)

\begin{tabular}{|c|c|c|c|c|c|c|}
\hline $\begin{array}{l}\text { Study (first author and } \\
\text { year) }\end{array}$ & $\begin{array}{l}\text { Functional } \\
\text { Test }\end{array}$ & Follow-Up & Outcomes & Main Results & $\begin{array}{l}\text { Risk of } \\
\text { Bias }\end{array}$ & $\begin{array}{l}\text { Level of } \\
\text { Evidence } \\
\text { (GRADE) }\end{array}$ \\
\hline & & months) & $\leq 300 \mathrm{~m}$ VS $>300 \mathrm{~m}$ & $5.76]^{* *}$ & & \\
\hline \multirow[t]{3}{*}{ Lee et al. [50], 2006.} & $6 \mathrm{MWT}$ & $36 \pm 12$ months & $\begin{array}{l}\text { Hospitalisation and } \\
\text { Mortality } \\
75-230 \text { m VS }>370 \text { m. }\end{array}$ & $\begin{array}{l}\mathrm{OR}=3.595 \% \mathrm{Cl}[1.1- \\
11.7]^{* *}\end{array}$ & Low & Low \\
\hline & & & $\begin{array}{l}\text { Hospitalisation and } \\
\text { Mortality } \\
231-310 m \text { VS }>370 m\end{array}$ & $\begin{array}{l}\mathrm{OR}=3.495 \% \mathrm{Cl}[1.01- \\
11.5]^{* *}\end{array}$ & & \\
\hline & & & $\begin{array}{l}\text { Hospitalisation and } \\
\text { Mortality } \\
311-370 m \text { VS }>370 m\end{array}$ & $\begin{array}{l}\mathrm{OR}=4.995 \% \mathrm{Cl}[1.5- \\
16.0]^{* *}\end{array}$ & & \\
\hline \multirow[t]{12}{*}{ Curtis et al. [51], 2004.} & $6 \mathrm{MWT}$ & 32 months & $\begin{array}{l}\text { All-Cause Mortality } \\
\leq 200 \mathrm{~m} \text { VS }>400 \mathrm{~m}\end{array}$ & $\begin{array}{l}\mathrm{HR}=1.5995 \% \mathrm{Cl}[0.88- \\
2.86]^{*}\end{array}$ & Low & Moderate \\
\hline & & & $\begin{array}{l}\text { All-Cause Mortality } \\
201-300 m \text { VS }>400 m\end{array}$ & $\begin{array}{l}\mathrm{HR}=1.0195 \% \mathrm{Cl}[0.57- \\
1.79]^{*}\end{array}$ & & \\
\hline & & & $\begin{array}{l}\text { All-Cause Mortality } \\
301-400 \mathrm{~m} \text { VS }>400 \mathrm{~m}\end{array}$ & $\begin{array}{l}\mathrm{HR}=1.1695 \% \mathrm{Cl}[0.72- \\
1.88]^{*}\end{array}$ & & \\
\hline & & & $\begin{array}{l}\text { HF Mortality } \\
\leq 200 \mathrm{~m} \text { VS }>400 \mathrm{~m}\end{array}$ & $\begin{array}{l}\mathrm{HR}=2.6295 \% \mathrm{Cl}[1.02- \\
6.74]^{* *}\end{array}$ & & Moderate \\
\hline & & & $\begin{array}{l}\text { HF Mortality } \\
201-300 \text { m VS }>400 m\end{array}$ & $\begin{array}{l}\mathrm{HR}=0.9395 \% \mathrm{Cl}[0.34- \\
2.55]^{*}\end{array}$ & & \\
\hline & & & $\begin{array}{l}\text { HF Mortality } \\
301-400 \text { m VS }>400 m\end{array}$ & $\begin{array}{l}\mathrm{HR}=0.86 \text { 95\%Cl [0.35- } \\
2.09]^{*}\end{array}$ & & \\
\hline & & & $\begin{array}{l}\text { All-Cause Hospitalisation } \\
\leq 200 m \text { VS }>400 m\end{array}$ & $\begin{array}{l}\mathrm{HR}=1.7695 \% \mathrm{Cl}[1.19- \\
2.60]^{* *}\end{array}$ & & Low \\
\hline & & & $\begin{array}{l}\text { All-Cause Hospitalisation } \\
201-300 m \text { VS }>400 m\end{array}$ & $\begin{array}{l}\mathrm{HR}=1.4195 \% \mathrm{Cl}[1.01- \\
1.99]^{* *}\end{array}$ & & \\
\hline & & & $\begin{array}{l}\text { All-Cause Hospitalisation } \\
301-400 \mathrm{~m} \text { VS }>400 \mathrm{~m}\end{array}$ & $\begin{array}{l}\mathrm{HR}=1.0995 \% \mathrm{Cl}[0.80- \\
1.47]^{*}\end{array}$ & & \\
\hline & & & $\begin{array}{l}\text { HF Hospitalisation } \\
\leq 200 m \text { VS }>400 m\end{array}$ & $\begin{array}{l}\mathrm{HR}=1.8495 \% \mathrm{Cl}[0.97- \\
3.49]^{*}\end{array}$ & & Low \\
\hline & & & $\begin{array}{l}\text { HF Hospitalisation } \\
201-300 m \text { VS }>400 m\end{array}$ & $\begin{array}{l}\mathrm{HR}=1.8495 \% \mathrm{Cl}[1.04- \\
3.29]^{* *}\end{array}$ & & \\
\hline & & & $\begin{array}{l}\text { HF Hospitalisation } \\
301-400 m \text { VS }>400 m\end{array}$ & $\begin{array}{l}\mathrm{HR}=1.4595 \% \mathrm{Cl}[0.85- \\
2.45]^{*}\end{array}$ & & \\
\hline Ingle et al. [52], 2014. & $6 \mathrm{MWT}$ & 5 years & $\begin{array}{l}\text { All-Cause Mortality } \\
\text { Per each } 10 \text { m increased. }\end{array}$ & $\begin{array}{l}\mathrm{HR}=0.98095 \% \mathrm{Cl}[0.974- \\
0.985]^{* * *}\end{array}$ & Low & Moderate \\
\hline \multirow[t]{3}{*}{ Alahdab et al. [53], 2009.} & $6 \mathrm{MWT}$ & 40 months-Mortality & $\begin{array}{l}\text { All-Cause Mortality } \\
\leq 200 \mathrm{~m} \text { VS }>200 \mathrm{~m}\end{array}$ & $\begin{array}{l}\mathrm{HR}=2.1495 \% \mathrm{Cl}[1.20- \\
3.81]^{* *}\end{array}$ & Low & Moderate \\
\hline & & 40 months-Mortality & $\begin{array}{l}\text { All-Cause Mortality } \\
\text { Per each } 1 \mathrm{~m} \text { increased }\end{array}$ & $\begin{array}{l}\mathrm{HR}=0.99895 \% \mathrm{Cl}[0.995- \\
0.999]^{* *}\end{array}$ & & \\
\hline & & $\begin{array}{l}18 \text { months-Hospitali- } \\
\text { zation }\end{array}$ & $\begin{array}{l}\text { HF Hospitalisation } \\
\leq 200 \mathrm{~m} \text { VS }>200 \mathrm{~m}\end{array}$ & $\begin{array}{l}\mathrm{HR}=1.62 \text { 95\%Cl [1.10- } \\
2.39]^{* *}\end{array}$ & & Low \\
\hline \multirow[t]{2}{*}{ Mangla et al. [54], 2013.} & $6 \mathrm{MWT}$ & 1080 days & $\begin{array}{l}\text { Hospitalisation and } \\
\text { Mortality } \\
\leq 189 \mathrm{~m} \text { VS }>189 \mathrm{~m} \\
\text { in HFpEF. }\end{array}$ & $\begin{array}{l}\mathrm{OR}=2.8195 \% \mathrm{Cl}[1.24- \\
6.40]^{* *}\end{array}$ & Low & Low \\
\hline & & & $\begin{array}{l}\text { Hospitalisation and } \\
\text { Mortality } \\
\leq 189 m \text { VS }>189 m \text { in } \\
\text { HFrEF. }\end{array}$ & $\begin{array}{l}\mathrm{OR}=1.9495 \% \mathrm{Cl}[1.30- \\
2.90]^{* *}\end{array}$ & & \\
\hline Hasin et al. [55], 2012. & $6 \mathrm{MWT}$ & $\begin{array}{l}\text { Median } 592 \text { days } \\
\text { (115-1453 days) }\end{array}$ & $\begin{array}{l}\text { All-Cause Mortality } \\
\text { Per } 10 \mathrm{~m} \text { walked short of } \\
300 \mathrm{~m}\end{array}$ & $\begin{array}{l}\mathrm{HR}=1.21195 \% \mathrm{Cl}[1.108- \\
1.322]^{* * *}\end{array}$ & Moderate & Moderate \\
\hline
\end{tabular}


Table 2 Outcomes, Results, Risk of Bias of Included Studies and Level of Evidence per Outcome according to GRADE Criteria (Continued)

\begin{tabular}{|c|c|c|c|c|c|c|}
\hline $\begin{array}{l}\text { Study (first author and } \\
\text { year) }\end{array}$ & $\begin{array}{l}\text { Functional } \\
\text { Test }\end{array}$ & Follow-Up & Outcomes & Main Results & $\begin{array}{l}\text { Risk of } \\
\text { Bias }\end{array}$ & $\begin{array}{l}\text { Level of } \\
\text { Evidence } \\
\text { (GRADE) }\end{array}$ \\
\hline \multirow[t]{2}{*}{ Passantino et al. [56], 2006.} & $6 \mathrm{MWT}$ & 23.9 months & $\begin{array}{l}\text { All-Cause Mortality } \\
<300 \mathrm{~m} \text { VS } \geq 300 \mathrm{~m}\end{array}$ & $\begin{array}{l}\mathrm{HR}=2.6695 \% \mathrm{Cl}[1.60- \\
4.42]^{* * *}\end{array}$ & Low & Moderate \\
\hline & & & $\begin{array}{l}\text { All-Cause Mortality } \\
\text { Per each } 70 \mathrm{~m} \text { decreased }\end{array}$ & $\begin{array}{l}\mathrm{HR}=2.0395 \% \mathrm{Cl}[1.29- \\
3.18]^{* *}\end{array}$ & & \\
\hline $\begin{array}{l}\text { Howie-Esquivel et al. [57], } \\
2008 .\end{array}$ & $6 \mathrm{MWT}$ & 90 days & $\begin{array}{l}\text { HF Hospitalisation } \\
>200 \mathrm{~m}\end{array}$ & $\begin{array}{l}\mathrm{HR}=0.9995 \% \mathrm{Cl}[0.99- \\
1.00]^{*}\end{array}$ & High & Low \\
\hline $\begin{array}{l}\text { Zotter-Tufaro et al. [58], } \\
2015 .\end{array}$ & $6 \mathrm{MWT}$ & $14.0 \pm 10.0$ months & $\begin{array}{l}\text { Hospitalisation and } \\
\text { Mortality } \\
>300 \mathrm{~m} \text { VS } \leq 300 \mathrm{~m}\end{array}$ & $\begin{array}{l}\mathrm{HR}=0.992 \text { 95\%Cl [0.990- } \\
0.995]^{* * *}\end{array}$ & Moderate & Low \\
\hline Boxer et al. [59], 2010. & $6 \mathrm{MWT}$ & 4 years & $\begin{array}{l}\text { All-Cause Mortality } \\
\text { Per each } 30 \text { m increased }\end{array}$ & $\begin{array}{l}\mathrm{HR}=0.8495 \% \mathrm{Cl}[0.74- \\
0.94]^{* *}\end{array}$ & Moderate & Moderate \\
\hline Ingle et al. [60], 2014. & $6 \mathrm{MWT}$ & 8 years & $\begin{array}{l}\text { All-Cause Mortality } \\
\text { Per each } 10 \mathrm{~m} \text { increased }\end{array}$ & $\begin{array}{l}\mathrm{HR}=0.98895 \% \mathrm{Cl}[0.981- \\
0.995]^{* * *}\end{array}$ & Low & Moderate \\
\hline Guazzi et al. [61], 2009. & $6 \mathrm{MWT}$ & $20.4 \pm 16.6$ months. & $\begin{array}{l}\text { Cardiac Mortality } \\
\text { Per each } 1 \mathrm{~m} \text { increased }\end{array}$ & $\begin{array}{l}\mathrm{HR}=0.99895 \% \mathrm{Cl}[0.995- \\
1.001]^{*}\end{array}$ & Low & Moderate \\
\hline McCabe et al. [62], 2017. & $6 \mathrm{MWT}$ & 30 days & $\begin{array}{l}\text { HF Hospitalisation } \\
\text { Per each } 30 \text { m increased }\end{array}$ & $\begin{array}{l}\mathrm{OR}=0.8495 \% \mathrm{Cl}[0.71- \\
0.99]^{* *}\end{array}$ & Moderate & Low \\
\hline \multirow[t]{4}{*}{ Vegh et al. [63], 2014.} & $6 \mathrm{MWT}$ & 3 years & $\begin{array}{l}\text { HF Hospitalisation } \\
\geq 350 \mathrm{~m} \text { VS }<280 \mathrm{~m}\end{array}$ & $\begin{array}{l}\mathrm{HR}=0.6195 \% \mathrm{Cl}[0.44- \\
0.85]^{* *}\end{array}$ & Moderate & Low \\
\hline & & & $\begin{array}{l}\text { Hospitalisation and } \\
\text { Mortality } \\
\geq 350 \mathrm{~m} \text { VS }<280 \mathrm{~m}\end{array}$ & $\begin{array}{l}\mathrm{HR}=0.5895 \% \mathrm{Cl}[0.43- \\
0.80]^{* * *}\end{array}$ & & Low \\
\hline & & & $\begin{array}{l}\text { HF Hospitalisation } \\
\geq 402 \mathrm{~m} \text { VS }<256 \mathrm{~m}\end{array}$ & $\begin{array}{l}\mathrm{HR}=0.6095 \% \mathrm{Cl}[0.44- \\
0.82]^{* * *}\end{array}$ & & Low \\
\hline & & & $\begin{array}{l}\text { Hospitalisation and } \\
\text { Mortality } \\
\geq 402 \mathrm{~m} \text { VS }<256 \mathrm{~m}\end{array}$ & $\begin{array}{l}\mathrm{HR}=0.5595 \% \mathrm{Cl}[0.43- \\
0.75]^{* * *}\end{array}$ & & Low \\
\hline Roul et al. [64], 1998. & 6MWT & 1000 days & $\begin{array}{l}\text { Hospitalisation and } \\
\text { Mortality } \\
\leq 300 \text { m VS > 300m }\end{array}$ & Log rank $=6.16^{* *}$ & Moderate & Low \\
\hline $\begin{array}{l}\text { Frankenstein et al. [65], } \\
2008 .\end{array}$ & $6 \mathrm{MWT}$ & $52.9 \pm 36.2$ months & $\begin{array}{l}\text { All-Cause Mortality } \\
\text { Per each } 1 \mathrm{~m} \text { increased }\end{array}$ & $\begin{array}{l}\mathrm{HR}=0.99695 \% \mathrm{Cl}[0.995- \\
0.997]^{* * *}\end{array}$ & Low & Moderate \\
\hline $\begin{array}{l}\text { Mene-Afejuku et al. [66], } \\
2017 .\end{array}$ & $6 \mathrm{MWT}$ & 6 months & $\begin{array}{l}\text { Hospitalisation and } \\
\text { Mortality } \\
314.66 \mathrm{~m} \pm 48.17 \mathrm{~m} \text { VS } \\
260.59 \mathrm{~m} \pm 66.65 \mathrm{~m}\end{array}$ & $\begin{array}{l}\mathrm{OR}=0.81995 \% \mathrm{Cl}[0.206- \\
3.257]^{*}\end{array}$ & Moderate & Low \\
\hline Ingle et al. [67], 2007. & $6 \mathrm{MWT}$ & $\begin{array}{l}36.6 \text { months } \\
\text { (28.2-45.0 months) }\end{array}$ & $\begin{array}{l}\text { All-Cause Mortality } \\
\text { Per each } 1 \mathrm{~m} \text { increased }\end{array}$ & $\begin{array}{l}\mathrm{HR}=0.99895 \% \mathrm{Cl}[0.996- \\
1.000]^{*}\end{array}$ & Low & Moderate \\
\hline Rostagno et al. [68], 2003. & $6 \mathrm{MWT}$ & 34 months & $\begin{array}{l}\text { All-Cause Mortality } \\
\text { Per each } 1 \mathrm{~m} \text { increased }\end{array}$ & $\begin{array}{l}\mathrm{HR}=0.99595 \% \mathrm{Cl}[0.993- \\
0.997]^{* * *}\end{array}$ & Low & Moderate \\
\hline Cahalin et al. [69], 1996. & $6 \mathrm{MWT}$ & $\begin{array}{l}62 \pm 45 \text { weeks }(1-183 \\
\text { weeks) }\end{array}$ & $\begin{array}{l}\text { Hospitalisation and } \\
\text { Mortality } \\
<300 \mathrm{~m} \text { VS } \geq 300 \mathrm{~m}\end{array}$ & $x^{2}=40 \%$ vs $12 \% * *$ & Moderate & Low \\
\hline $\begin{array}{l}\text { Frankenstein et al. [70], } \\
2008 .\end{array}$ & 6MWT & $\begin{array}{l}42 \text { months ( } 22-80 \\
\text { months) }\end{array}$ & $\begin{array}{l}\text { All-Cause Mortality } \\
\text { Per each } 1 \mathrm{~m} \text { increased }\end{array}$ & $\begin{array}{l}\mathrm{HR}=0.99695 \% \mathrm{Cl}[0.995- \\
0.997]^{* *}\end{array}$ & Moderate & Moderate \\
\hline Rubim et al. [71], 2006. & $6 \mathrm{MWT}$ & $\begin{array}{l}18 \text { months (12-24 } \\
\text { months) }\end{array}$ & $\begin{array}{l}\text { All-Cause Mortality } \\
\geq 520 \mathrm{~m} \text { VS }<520 \mathrm{~m}\end{array}$ & $\begin{array}{l}\mathrm{OR}=-0.008195 \% \mathrm{Cl} \\
{[0.0029-0.0133]^{* * *}}\end{array}$ & Low & Moderate \\
\hline Kanagala et al. [72], 2019. & $6 \mathrm{MWT}$ & $\begin{array}{l}1429 \text { days ( } 1157- \\
1657 \text { days) }\end{array}$ & $\begin{array}{l}\text { Hospitalisation and } \\
\text { Mortality } \\
\text { Per each } 1 \mathrm{~m} \text { increased }\end{array}$ & $\begin{array}{l}\mathrm{HR}=0.65995 \% \mathrm{Cl}[0.465- \\
0.934]^{* *}\end{array}$ & Low & Low \\
\hline Zugck et al. [73], 2001. & $6 \mathrm{MWT}$ & $28.3 \pm 14.1$ months & $\begin{array}{l}\text { All-Cause Mortality } \\
\text { Per each } 1 \mathrm{~m} \text { increased }\end{array}$ & $\begin{array}{l}\mathrm{HR}=0.9995 \% \mathrm{Cl}[0.98- \\
0.99]^{* *}\end{array}$ & Moderate & Moderate \\
\hline Cahalin et al. [74], 2013. & $6 \mathrm{MWT}$ & $22.8 \pm 22.1$ months & Cardiac Mortality & $\mathrm{HR}=0.9995 \% \mathrm{Cl}[0.99-$ & Low & Moderate \\
\hline
\end{tabular}


Table 2 Outcomes, Results, Risk of Bias of Included Studies and Level of Evidence per Outcome according to GRADE Criteria (Continued)

\begin{tabular}{|c|c|c|c|c|c|c|}
\hline $\begin{array}{l}\text { Study (first author and } \\
\text { year) }\end{array}$ & $\begin{array}{l}\text { Functional } \\
\text { Test }\end{array}$ & Follow-Up & Outcomes & Main Results & $\begin{array}{l}\text { Risk of } \\
\text { Bias }\end{array}$ & $\begin{array}{l}\text { Level of } \\
\text { Evidence } \\
\text { (GRADE) }\end{array}$ \\
\hline & & & Per each $1 \mathrm{~m}$ increased & $0.99]^{* *}$ & & \\
\hline & & & $\begin{array}{l}\text { Cardiac Mortality } \\
>300 \mathrm{~m} \text { VS } \leq 300 \mathrm{~m}\end{array}$ & $\begin{array}{l}\mathrm{HR}=0.1895 \% \mathrm{Cl}[0.04- \\
0.89]^{* *}\end{array}$ & & \\
\hline Reibis et al. [75], 2010. & 6MWT & $731 \pm 215$ days & $\begin{array}{l}\text { All-Cause Mortality } \\
\text { Per each } 50 \text { m increasd }\end{array}$ & $\begin{array}{l}H R=0.9395 \% \mathrm{Cl}[0.86- \\
1.00]^{* *}\end{array}$ & Low & Moderate \\
\hline \multirow[t]{3}{*}{ Castel et al. [76], 2009.} & $6 \mathrm{MWT}$ & $24.4 \pm 18.1$ months & $\begin{array}{l}\text { Cardiac Mortality } \\
<225 \mathrm{~m} \text { VS }>400 \mathrm{~m}\end{array}$ & $\begin{array}{l}\mathrm{HR}=5.6095 \% \mathrm{Cl}[1.23- \\
25.30]^{* *}\end{array}$ & Low & Moderate \\
\hline & & & $\begin{array}{l}\text { Cardiac Mortality } \\
225-310 m \text { VS }>400 m\end{array}$ & $\begin{array}{l}\mathrm{HR}=1.2895 \% \mathrm{Cl}[0.23- \\
7.08]^{*}\end{array}$ & & \\
\hline & & & $\begin{array}{l}\text { Cardiac Mortality } \\
310-400 m \text { VS }>400 m\end{array}$ & $\begin{array}{l}\mathrm{HR}=4.1095 \% \mathrm{Cl}[0.79- \\
21.52]^{*}\end{array}$ & & \\
\hline Kamiya et al. [77], 2017. & $6 \mathrm{MWT}$ & $2.3 \pm 1.9$ years & $\begin{array}{l}\text { All-Cause Mortality } \\
\text { Per each } 10 \mathrm{~m} \text { increased }\end{array}$ & $\begin{array}{l}H R=0.9695 \% \mathrm{Cl}[0.94- \\
0.97]^{* * *}\end{array}$ & Low & Moderate \\
\hline \multirow[t]{3}{*}{ García et al. [78], 2019.} & SPPB & 1 year & $\begin{array}{l}\text { HF Hospitalisation } \\
\mathrm{SPPB} \leq 7 \mathrm{VS} S \mathrm{SPB}>7\end{array}$ & $\begin{array}{l}\mathrm{OR}=6.795 \% \mathrm{Cl}[1.5- \\
30.4]^{* *}\end{array}$ & Moderate & $\begin{array}{l}\text { Not } \\
\text { Reported }\end{array}$ \\
\hline & & & $\begin{array}{l}\text { All-Cause Mortality } \\
\mathrm{SPPB} \leq 7 \mathrm{VS} \mathrm{SPPB}>7\end{array}$ & $\mathrm{OR}=1.295 \% \mathrm{Cl}[0.3-5.4]^{*}$ & & Very Low \\
\hline & & & $\begin{array}{l}\text { Hospitalisation and } \\
\text { Mortality } \\
\text { SPPB } \leq 7 \text { VS SPPB }>7\end{array}$ & $\begin{array}{l}\mathrm{OR}=3.695 \% \mathrm{Cl}[1.0- \\
12.9]^{* *}\end{array}$ & & Very Low \\
\hline \multirow[t]{3}{*}{ Hornsby et al. [79], 2019.} & SPPB & 6 months & $\begin{array}{l}\text { Hospitalisation and } \\
\text { Mortality } \\
\text { Per 1-unit change in SPPB }\end{array}$ & $\begin{array}{l}\mathrm{OR}=0.8195 \% \mathrm{Cl}[0.69- \\
0.94]^{* *}\end{array}$ & Moderate & Very Low \\
\hline & & & $\begin{array}{l}\text { Number of All-Cause } \\
\text { Hospitalisations } \\
\text { Per 1-unit change in SPPB }\end{array}$ & $\begin{array}{l}\mathrm{IRR}=0.9295 \% \mathrm{Cl}[0.86- \\
0.97]^{* *}\end{array}$ & & $\begin{array}{l}\text { Not } \\
\text { Reported }\end{array}$ \\
\hline & & & $\begin{array}{l}\text { Days Hospitalized or } \\
\text { Dead } \\
\text { Per 1-unit change in SPPB }\end{array}$ & $\begin{array}{l}\mathrm{IRR}=0.8595 \% \mathrm{Cl}[0.73- \\
0.99]^{* *}\end{array}$ & & $\begin{array}{l}\text { Not } \\
\text { Reported }\end{array}$ \\
\hline \multirow[t]{3}{*}{ Chiarantini et al. [80], 2010.} & SPPB & $\begin{array}{l}30 \text { months (median } \\
444 \text { days) }\end{array}$ & $\begin{array}{l}\text { All-Cause Mortality } \\
\text { SPPB } 0 \text { VS SPPB 9-12 }\end{array}$ & $\begin{array}{l}\mathrm{HR}=6.0695 \% \mathrm{Cl}[2.19- \\
16.76]^{* * *}\end{array}$ & Moderate & Very Low \\
\hline & & & $\begin{array}{l}\text { All-Cause Mortality } \\
\text { SPPB 1-4 VS SPPB 9-12 }\end{array}$ & $\begin{array}{l}\mathrm{HR}=4.7895 \% \mathrm{Cl}[1.63- \\
14.02]^{* *}\end{array}$ & & \\
\hline & & & $\begin{array}{l}\text { All-Cause Mortality } \\
\text { SPPB 5-8 VS SPPB 9-12 }\end{array}$ & $\begin{array}{l}\mathrm{HR}=1.9595 \% \mathrm{Cl}[0.67- \\
5.70]^{*}\end{array}$ & & \\
\hline Zaharias et al. [81], 2014. & SPPB & 3 months & $\begin{array}{l}\text { Hospitalisation and } \\
\text { Mortality } \\
\text { Per each } 1 \text { point decreased }\end{array}$ & $\begin{array}{l}\mathrm{HR}=1.04295 \% \mathrm{Cl}[0.89- \\
1.23]^{*}\end{array}$ & Moderate & Very Low \\
\hline Lo et al. [82], 2015. & Gait Speed & 10 years & $\begin{array}{l}\text { All-Cause Mortality } \\
<0.8 \mathrm{~m} / \mathrm{s} \text { VS } \geq 0.8 \mathrm{~m} / \mathrm{s}\end{array}$ & $\begin{array}{l}\mathrm{HR}=1.3795 \% \mathrm{Cl}[1.10- \\
1.70]^{* *}\end{array}$ & Low & Low \\
\hline \multirow[t]{3}{*}{ Pulignano et al. [83], 2016.} & Gait Speed & 1 year & $\begin{array}{l}\text { All-Cause Mortality } \\
\text { Gait speed (tertiles) }\end{array}$ & $\begin{array}{l}\mathrm{HR}=0.62095 \% \mathrm{Cl}[0.434- \\
0.884]^{* *}\end{array}$ & Low & Low \\
\hline & & & $\begin{array}{l}\text { HF Hospitalisation } \\
\text { Gait speed (tertiles) }\end{array}$ & $\begin{array}{l}\mathrm{OR}=0.69795 \% \mathrm{Cl}[0.547- \\
0.899]^{* *}\end{array}$ & & Low \\
\hline & & & $\begin{array}{l}\text { All-Cause Hospitalisation } \\
\text { Gait speed (tertiles) }\end{array}$ & $\begin{array}{l}\mathrm{HR}=0.74195 \% \mathrm{Cl}[0.613- \\
0.895]^{* *}\end{array}$ & & Low \\
\hline \multirow[t]{2}{*}{ Chaudhry et al. [84], 2013.} & Gait Speed & 20 years & $\begin{array}{l}\text { All-Cause Hospitalisation } \\
\leq 0.8 \mathrm{~m} / \mathrm{s} \mathrm{VS}>0.8 \mathrm{~m} / \mathrm{s}\end{array}$ & $\begin{array}{l}\mathrm{HR}=1.2895 \% \mathrm{Cl}[1.06- \\
1.55]^{* *}\end{array}$ & Low & Low \\
\hline & & & $\begin{array}{l}\text { Hospitalisation and } \\
\text { Mortality } \\
\leq 0.8 \mathrm{~m} / \mathrm{s} \vee \mathrm{S}>0.8 \mathrm{~m} / \mathrm{s}\end{array}$ & $\begin{array}{l}\mathrm{HR}=1.3195 \% \mathrm{Cl}[1.08- \\
1.58]^{* *}\end{array}$ & & Low \\
\hline Tanaka et al. [85], 2018. & Gait Speed & $1.7 \pm 0.5$ years & All-Cause Mortality & $\mathrm{HR}=0.8095 \% \mathrm{Cl}[0.37-$ & Moderate & Low \\
\hline
\end{tabular}


Table 2 Outcomes, Results, Risk of Bias of Included Studies and Level of Evidence per Outcome according to GRADE Criteria (Continued)

\begin{tabular}{|c|c|c|c|c|c|c|}
\hline $\begin{array}{l}\text { Study (first author and } \\
\text { year) }\end{array}$ & $\begin{array}{l}\text { Functional } \\
\text { Test }\end{array}$ & Follow-Up & Outcomes & Main Results & $\begin{array}{l}\text { Risk of } \\
\text { Bias }\end{array}$ & $\begin{array}{l}\text { Level of } \\
\text { Evidence } \\
\text { (GRADE) }\end{array}$ \\
\hline & & & $1.0-1.14 \mathrm{~m} / \mathrm{s}$ VS $>1.14 \mathrm{~m} / \mathrm{s}$ & $1.74]^{*}$ & & \\
\hline & & & $\begin{array}{l}\text { All-Cause Mortality } \\
0.82-0.99 \mathrm{~m} / \mathrm{s} \text { VS }>1.14 \mathrm{~m} / \mathrm{s}\end{array}$ & $\begin{array}{l}\mathrm{HR}=1.4695 \% \mathrm{Cl}[0.75- \\
2.83]^{*}\end{array}$ & & \\
\hline & & & $\begin{array}{l}\text { All-Cause Mortality } \\
<0.82 \mathrm{~m} / \mathrm{s} \text { VS }>1.14 \mathrm{~m} / \mathrm{s}\end{array}$ & $\begin{array}{l}\mathrm{HR}=2.6595 \% \mathrm{Cl}[1.35- \\
5.20]^{* *}\end{array}$ & & \\
\hline \multirow[t]{3}{*}{ Tanaka et al. [86], 2019.} & Gait Speed & $2.1 \pm 1.9$ years & $\begin{array}{l}\text { All-Cause Mortality } \\
\text { Per each } 0.1 \mathrm{~m} / \mathrm{s} \text { increased }\end{array}$ & $\begin{array}{l}\mathrm{HR}=0.8395 \% \mathrm{Cl}[0.73- \\
0.95]^{* *}\end{array}$ & Low & Low \\
\hline & & & $\begin{array}{l}\text { HF Hospitalisation } \\
\text { Per each } 0.1 \mathrm{~m} / \mathrm{s} \text { increased }\end{array}$ & $\begin{array}{l}\mathrm{HR}=0.9195 \% \mathrm{Cl}[0.83- \\
0.99]^{* *}\end{array}$ & & Low \\
\hline & & & $\begin{array}{l}\text { Hospitalisation and } \\
\text { Mortality } \\
\text { Per each } 0.1 \mathrm{~m} / \mathrm{s} \text { increased }\end{array}$ & $\begin{array}{l}\mathrm{HR}=0.9095 \% \mathrm{Cl}[0.83- \\
0.97]^{* *}\end{array}$ & & Low \\
\hline \multirow[t]{2}{*}{$\begin{array}{l}\text { Rodríguez-Pascual et al. } \\
\text { [87], } 2017 .\end{array}$} & Gait Speed & 1 year & $\begin{array}{l}\text { All-Cause Mortality } \\
\mathrm{GS}<0.65 \mathrm{~m} / \mathrm{s} \text { VS GS } \geq 0.65 \\
\mathrm{~m} / \mathrm{s}\end{array}$ & $\begin{array}{l}\mathrm{HR}=1.8695 \% \mathrm{Cl}[0.95- \\
3.65]^{*}\end{array}$ & Low & Low \\
\hline & & & $\begin{array}{l}\text { All-Cause Hospitalisation } \\
\mathrm{GS}<0.65 \mathrm{~m} / \mathrm{s} \text { VS GS } \geq 0.65 \\
\mathrm{~m} / \mathrm{s}\end{array}$ & $\begin{array}{l}\mathrm{HR}=1.5795 \% \mathrm{Cl}[0.98- \\
2.52]^{*}\end{array}$ & & Low \\
\hline \multirow[t]{2}{*}{ Vidán et al. [88], 2016.} & Gait Speed & 1 year & $\begin{array}{l}\text { All-Cause Mortality } \\
\text { GS }<0.65 \mathrm{~m} / \mathrm{s} \text { VS GS } \geq 0.65 \\
\mathrm{~m} / \mathrm{s}\end{array}$ & $\begin{array}{l}\mathrm{HR}=1.4895 \% \mathrm{Cl}[0.95- \\
2.32]^{*}\end{array}$ & Low & Low \\
\hline & & & $\begin{array}{l}\text { All-Cause Hospitalisation } \\
\mathrm{GS}<0.65 \mathrm{~m} / \mathrm{s} \text { VS GS } \geq 0.65 \\
\mathrm{~m} / \mathrm{s}\end{array}$ & $\begin{array}{l}\mathrm{OR}=1.6795 \% \mathrm{Cl}[0.98- \\
2.85]^{*}\end{array}$ & & Low \\
\hline Kamiya et al. [77], 2017. & Gait Speed & $2.3 \pm 1.9$ years & $\begin{array}{l}\text { All-Cause Mortality } \\
\text { Per each } 0.1 \mathrm{~m} / \mathrm{s} \text { increased }\end{array}$ & $\begin{array}{l}\mathrm{HR}=0.8795 \% \mathrm{Cl}[0.81- \\
0.93]^{* * *}\end{array}$ & Low & Low \\
\hline
\end{tabular}

6MWT Six Minutes Walking Test. $m$ Meters. HF Heart Failure. HR Hazard Ratio. Cl Confidence Interval. OR: Odds Ratio. $X^{2}$ : Chi-square test. HFrEF Patients with Heart Failure with Reduced Ejection Fraction (Systolic Heart Failure). HFPEF Patients with Heart Failure with Preserved Ejection Fraction (Diastolic Heart Failure). SPPB Short Physical Performance Battery. GS Gait Speed. IRR Incidence Rate Ratio. * $p>0.05 .{ }^{* *} p<0.05 .{ }^{* * *} p<0.001$

hospitalisation and mortality for any cause $(\mathrm{HR}=1.31$ 95\%CI [1.08-1.58, $p<0.05]$ ) [84].

\section{Physical functional performance and hospitalisation}

Patients with HFrEF with poor physical performance in the 6MWT showed a larger risk of All-Cause of Hospitalisation $[\mathrm{OR}=14.02 \quad 95 \% \mathrm{CI} \quad(4.90-40.14), p=0.001]$ [48] as patients with HFrEF and HFpEF $[\mathrm{HR}=1.41$ 95\%CI (1.01-1.99), $p<0.05$ ] [51]. A score below 7 points on the SPPB was also associated with a larger risk of HF Hospitalisation (OR $=6.7$ 95\%CI [1.5-30.4, $p<0.05])$ in patients with acute HF [78].

\section{Risk of Bias assessment}

The risk of bias of included observational longitudinal cohort studies is shown in Table 3. In summary, 26 studies $(59.10 \%)$ reported a low risk of bias, and 17 studies $(38,63 \%)$ showed a moderate risk of bias. Selection bias $(97,72 \%)$ were usual across the included studies. Using GRADE criteria, observational longitudinal cohort studies reported a low evidence in most of the prognostic outcomes. However, HF mortality and all-cause mortality showed a moderate evidence in the 6-MWT (Table 4).

\section{Discussion}

\section{Main findings and comparison with other studies}

The current systematic review and meta-analysis showed that patients with HFrEF and HFpEF who reported a poor physical functional performance in 6-MWT have an increased risk of all-cause of mortality and an increased risk of HF mortality. There was consistency in the risk of all-cause of mortality and HF mortality between the studies included in each meta-analysis (Fig. 2a and Fig. 2b) and the GRADE criteria also reported a moderate level of evidence per otucome. Although patients with HFrEF who decreased the meters they walked in the 6MWT during follow-up showed an increased risk of all-cause of mortality, there was no decreased risk of all-cause of mortality between patients with HFrEF and HFpEF who increased the meters they walked in the 6MWT during follow-up [52, 53, 59, 60, 65, 67, 68, 70, $73,75,77]$. Maybe this is beacuse the most of included studies in the meta-analysis reported a decreased risk of 


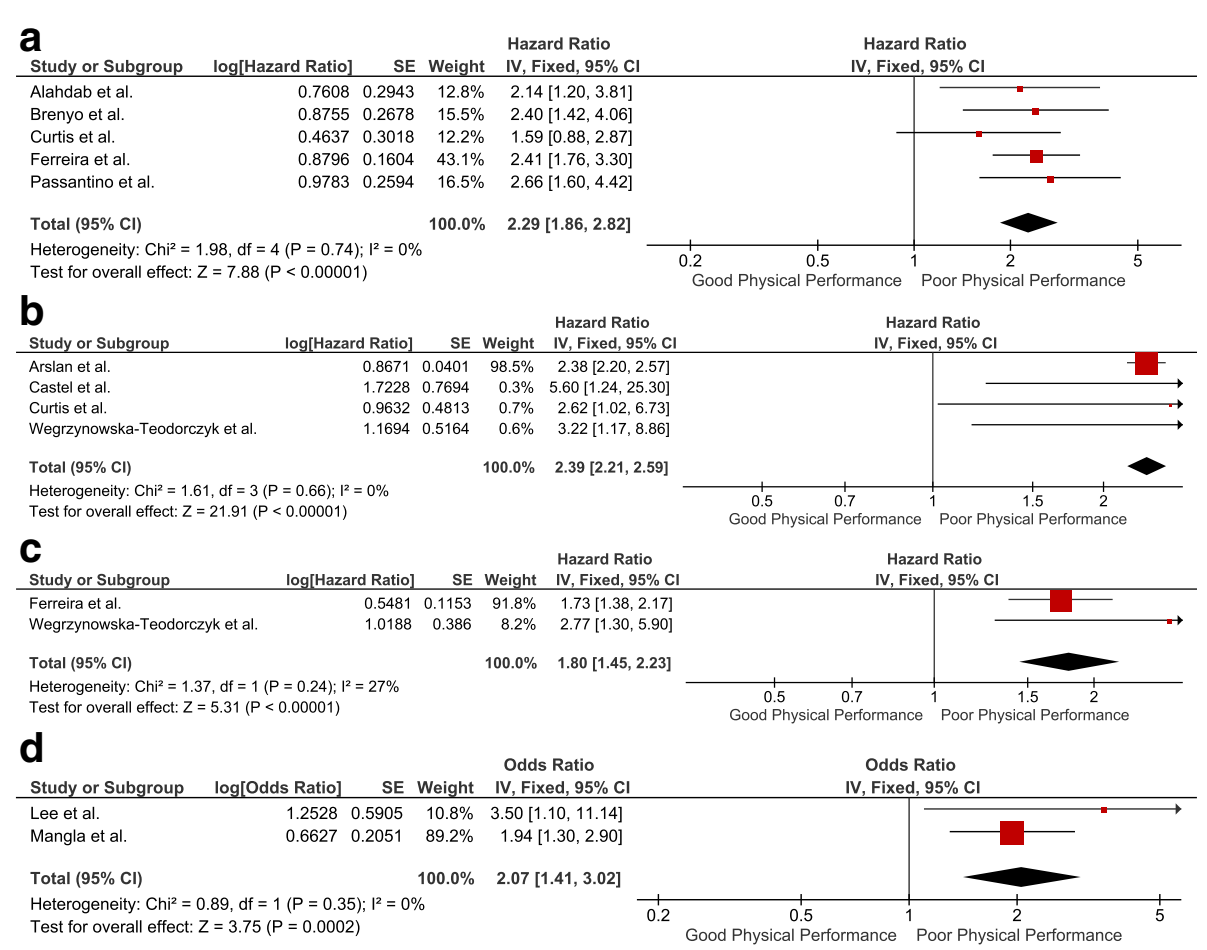

Fig. 2 Forest Plots ilustrating the risk of All-Cause Mortality (a), the risk of HF Mortality (b) and the risk of the combined endpoint of Hospitalisation and Mortality for any cause (c and d) in the 6MWT. Patients with Poor Physical Functional Performance Versus Patients with Good Physical Functional Performance

mortality for every $1 \mathrm{~m}$ increased [53, 65, 67, 68, 70, 73] or every $10 \mathrm{~m}[52,60,77]$ increased, while a systematic review determined that $45 \mathrm{~m}$ is the clinically meaningful change in the 6MWT [89]. Patients with HF who showed a poor physical functional performance in the $6 \mathrm{MWT}$ also reported an increased risk of the combined endpoint of hospitalisation and mortality for any cause (Fig. 2c and Fig. 2d), an increased risk of HF hospitalisation (Additional file 5) and an increased risk of all-cause of hospitalisation [48, 51]. However, the level of evidence of those outcomes was low according to the GRADE criteria. Moreover, there was a lack of homogeneity regarding which cut-off point should be used to stratify patients with HF based on their physical functional performance in the 6MWT. A distance traveled $<300 \mathrm{~m}$ was the most used distance to define patients with poor physical performance in the $6 \mathrm{MWT}$ in this study [47,
$49,55,56,58,59,61,62,64,69,74]$, while a previous review reported that a distance traveled $\leq 350 \mathrm{~m}$ in 6 MWT could be the most indicative distance of poor physical functional performance and worse prognosis in patients with HF [24].

A score between 1 and 4 points on the SPPB was associated with an increased risk of all-cause of mortality in this systematic review [80]. However, in the current study a score below 7 points on the SPPB seems to be the most indicative of a worse prognosis in patients with HF since it was associated with a larger risk of the combined endpoint of hospitalisation and mortality for any cause and a larger risk of HF hospitalisation [78]. GRADE criteria showed a very low level of evidence per outcome in each outcome examined by the SPPB. Moreover, meta-analysis on physical functional performance on the SPPB and prognosis in patients with HF could

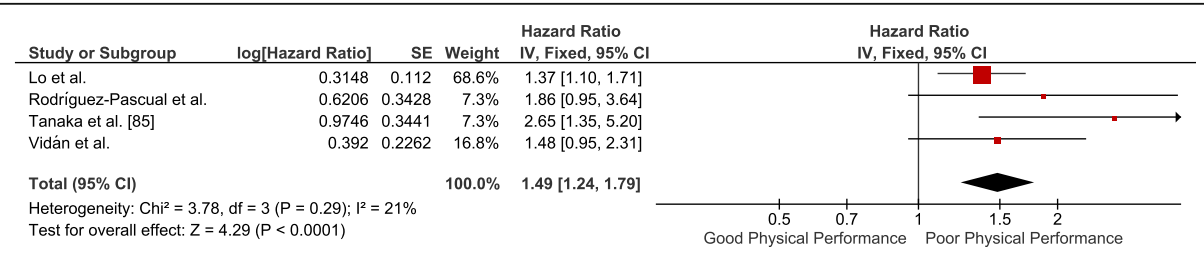

Fig. 3 Forest Plot ilustrating the risk of All-Cause Mortality in the Gait Speed Test. Patients with slower Gait Speed Versus Patients with faster Gait Speed 
Table 3 Risk of Bias Assessment of Cohort Studies (The Newcastle Ottawa Scale (NOS)).

\begin{tabular}{|c|c|c|c|c|c|c|c|c|c|c|c|c|}
\hline \multirow[t]{2}{*}{ First Author } & \multicolumn{4}{|c|}{ Selection } & \multicolumn{2}{|c|}{ Comparability } & \multicolumn{4}{|c|}{ Outcome } & \multirow{2}{*}{$\begin{array}{l}\text { Total } \\
\text { Score }\end{array}$} & \multirow[t]{2}{*}{$\begin{array}{l}\text { Quality } \\
\end{array}$} \\
\hline & 1 & 2 & 3 & 4 & 5 & 6 & & & 8 & 9 & & \\
\hline Brenyo et al. [45] & + & + & + & & + & + & & & + & + & $7 / 9$ & $\mathbf{L}$ \\
\hline $\begin{array}{l}\text { Ferreira et al. } \\
\text { [46] }\end{array}$ & + & + & + & & + & + & & & & & $6 / 9$ & M \\
\hline $\begin{array}{l}\text { Wegrzynowska- } \\
\text { Teodorccyk et al. } \\
{[47]}\end{array}$ & - & + & + & & + & + & & & + & + & $7 / 9$ & L \\
\hline Bittner et al. [48] & + & + & + & & + & + & & & - & + & 7/9 & L \\
\hline Arslan et al. [49] & - & + & + & & + & + & & & + & - & $5 / 9$ & M \\
\hline Lee et al. [50] & + & + & + & & + & + & & & + & + & $8 / 9$ & $\mathbf{L}$ \\
\hline Curtis et al. [51] & + & + & + & & + & + & & & + & + & $8 / 9$ & $\mathbf{L}$ \\
\hline Ingle et al. [52] & + & + & + & & + & + & & & + & + & $7 / 9$ & L \\
\hline $\begin{array}{c}\text { Alahdab et al. } \\
\text { [53] }\end{array}$ & - & + & + & & + & + & & & + & + & $7 / 9$ & $\mathbf{L}$ \\
\hline $\begin{array}{c}\text { Mangla et al. } \\
\text { [54] }\end{array}$ & + & + & + & & + & + & & & + & + & $8 / 9$ & L \\
\hline Hasin et al. [55] & & + & + & & + & + & & & + & - & $6 / 9$ & M \\
\hline $\begin{array}{l}\text { Passantino et al. } \\
\text { [56] }\end{array}$ & + & + & + & & - & + & & & + & + & $7 / 9$ & L \\
\hline $\begin{array}{l}\text { Howie-Esquivel } \\
\text { et al. [57] }\end{array}$ & - & + & + & & - & - & & & - & + & $3 / 9$ & H \\
\hline $\begin{array}{l}\text { Zotter-Tufaro et } \\
\text { al. [58] }\end{array}$ & - & + & + & & - & + & & & + & + & $6 / 9$ & M \\
\hline Boxer et al. [59] & & + & + & & + & + & & & + & - & $6 / 9$ & M \\
\hline Ingle et al. [60] & $T$ & + & + & & + & + & & & + & + & $7 / 9$ & L \\
\hline Guazzi et al. [61] & + & + & + & & - & + & & & + & + & $7 / 9$ & L \\
\hline $\begin{array}{l}\text { McCabe et al. } \\
\text { [62] }\end{array}$ & - & + & + & & + & + & & & - & + & $6 / 9$ & M \\
\hline Vegh et al. [63] & - & + & + & & + & + & & & + & - & $6 / 9$ & M \\
\hline Roul et al. [64] & & + & + & & $T$ & + & & & + & + & $6 / 9$ & M \\
\hline $\begin{array}{l}\text { Frankenstein et } \\
\text { al. [65] }\end{array}$ & + & + & + & & + & + & & & + & + & $8 / 9$ & L \\
\hline $\begin{array}{l}\text { Mene-Afejuku et } \\
\text { al. [66] }\end{array}$ & - & + & + & & - & + & & & - & + & $5 / 9$ & M \\
\hline Ingle et al. [67] & + & + & + & & + & + & & & + & + & $7 / 9$ & L \\
\hline $\begin{array}{l}\text { Rostagno et al. } \\
{[68]}\end{array}$ & + & + & + & & + & + & & & + & + & $8 / 9$ & $\mathbf{L}$ \\
\hline $\begin{array}{c}\text { Cahalin et al. } \\
\text { [69] }\end{array}$ & - & + & + & & - & - & & & + & + & $5 / 9$ & M \\
\hline $\begin{array}{l}\text { Frankenstein et } \\
\text { al. [70] }\end{array}$ & + & + & + & & - & - & & & + & + & $6 / 9$ & M \\
\hline Rubim et al. [71] & + & + & + & & + & + & & & + & + & $7 / 9$ & L \\
\hline $\begin{array}{l}\text { Kanagala et al. } \\
\text { [72] }\end{array}$ & & + & + & & + & + & & & + & + & $7 / 9$ & $\mathbf{L}$ \\
\hline Zugck et al. [73] & & + & + & & - & + & & & + & + & $6 / 9$ & M \\
\hline $\begin{array}{l}\text { Cahalin et al. } \\
\text { [74] }\end{array}$ & + & + & + & - & + & + & & & + & + & $8 / 9$ & L \\
\hline Reibis et al. [75] & + & + & + & & + & + & & & + & + & $8 / 9$ & $\mathbf{L}$ \\
\hline Castel et al. [76] & & + & + & & + & + & & & + & + & $7 / 9$ & $\mathbf{L}$ \\
\hline $\begin{array}{c}\text { Kamiya et al. } \\
\text { [77] }\end{array}$ & + & + & + & - & + & + & & & + & + & $8 / 9$ & L \\
\hline García et al. [78] & - & + & + & & + & + & & & + & & $5 / 9$ & $\mathbf{M}$ \\
\hline $\begin{array}{l}\text { Hornsby et al. } \\
\text { [79] }\end{array}$ & & + & + & & + & + & & & & + & $6 / 9$ & M \\
\hline $\begin{array}{c}\text { Chiarantini et al. } \\
{[80]}\end{array}$ & - & + & + & - & + & + & & & + & - & $6 / 9$ & M \\
\hline $\begin{array}{l}\text { Zaharias et al. } \\
\text { [81] }\end{array}$ & - & + & + & - & - & - & & & - & + & $4 / 9$ & M \\
\hline Lo et al. [82] & + & + & + & + & + & + & & & + & + & 9/9 & $\mathbf{L}$ \\
\hline 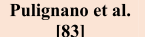 & + & + & + & & + & + & & & + & + & 8/9 & $\mathbf{L}$ \\
\hline Chaudhry et al. & + & + & + & - & + & + & & & + & - & $7 / 9$ & $\mathbf{L}$ \\
\hline $\begin{array}{c}\text { Tanaka et al. } \\
\text { [85] }\end{array}$ & + & + & + & & + & - & & & + & + & $6 / 9$ & M \\
\hline $\begin{array}{c}\text { Tanaka et al. } \\
{[86]}\end{array}$ & + & + & + & & - & + & & & + & + & $7 / 9$ & L \\
\hline $\begin{array}{c}\text { Rodriguez- } \\
\text { Pascual et al. } \\
\text { [87] }\end{array}$ & + & + & + & - & - & + & & & + & + & $7 / 9$ & L \\
\hline Vidán et al. [88] & + & + & + & & + & + & & - & + & + & $8 / 9$ & L \\
\hline
\end{tabular}

Note: The NOS assigns up to a maximum of nine points for the least risk of bias based on 3 domains: selection of study groups (four points); comparability of groups (two points); and ascertainment of exposure and outcomes (three points). This checklist has been recommended for cohort studies. The risk of bias based on the NOS was classified as: Low Risk of Bias (7-9 points), Moderate Risk of Bias (4-6 points) and High Risk of Bias (0-3 points). Abbreviations: Quality: High Risk of Bias (H); Moderate Risk of Bias (M); Low Risk of Bias (L); NOTE. Newcastle-Ottawa Quality Assessment Scale: cohort studies: $1=$ Representativeness of the exposed cohort; 2 = Selection of the non-exposed cohort; $3=$ Ascertainment of exposure; $4=$ Demonstration that outcome of interest was not present at start of study; $5-6=$ Comparability of cohorts on the basis of the design or analysis; 7 = Assessment of outcome; $8=$ Was follow-up long enough for outcomes to occur; $9=$ Adequacy of follow-up of cohorts

not be performed. As the present review, a score below 7 points on the SPPB was also associated with large risk of all-cause mortality in older adults [90]. However, other studies reported a large risk of mortality or hospitalisation in older adults who showed a score below 5 points [80, 91-93].
Patients who showed a slower gait speed also reported an increased risk of all-cause of mortality (Fig. 3), above all, when gait speed was slower than $0.65 \mathrm{~m} / \mathrm{s}$ (Additional file 5). Moreover, patients with HF who showed a slower gait speed also reported an increased risk of allcause of hospitalisation (Additional file 5) and an 
Table 4 Summary of Findings and Quality of Evidence Assessment of Included Observational Longitudinal Cohort Studies (GRADE)

\begin{tabular}{|c|c|c|c|c|c|c|c|c|c|c|}
\hline \multicolumn{5}{|c|}{ Summary of findings } & \multicolumn{6}{|c|}{ Quality of evidence assessment (GRADE) } \\
\hline Outcomes & $\begin{array}{l}\mathrm{N}^{\circ} \\
\text { studies }\end{array}$ & $\begin{array}{l}\mathrm{N}^{\circ} \\
\text { participants }\end{array}$ & Design $^{a}$ & $\begin{array}{l}\text { Risk of } \\
\text { Bias }^{\text {b }}\end{array}$ & Inconsistency $^{c}$ & $\underset{d}{\text { Indirectness }}$ & $\operatorname{Imprecision}^{\mathrm{e}}$ & Other & $\begin{array}{l}\text { Level of } \\
\text { Evidence }\end{array}$ & Importance \\
\hline \multicolumn{11}{|c|}{ Six Minutes Walking Test (6-MWT) } \\
\hline $\begin{array}{l}\text { All-Cause } \\
\text { Mortality }\end{array}$ & 18 & 15,033 & Observational & NO & $\begin{array}{l}\text { Consistency (+ } \\
\text { 1) }\end{array}$ & NO & NO & NO & Moderate & Critical \\
\hline $\begin{array}{l}\text { All-Cause } \\
\text { Hospitalisation }\end{array}$ & 2 & 1374 & Observational & NO & Not Serious & NO & NO & NO & Low & Critical \\
\hline HF Mortality & 6 & 1493 & Observational & NO & $\begin{array}{l}\text { Consistency (+ } \\
\text { 1) }\end{array}$ & NO & NO & NO & Moderate & Critical \\
\hline $\begin{array}{l}\text { HF } \\
\text { Hospitalisation }\end{array}$ & 6 & 1851 & Observational & $\begin{array}{l}\text { Not } \\
\text { Serious }\end{array}$ & Not Serious & NO & Not Serious & NO & Low & Critical \\
\hline $\begin{array}{l}\text { Hospitalisation } \\
\text { and Mortality }\end{array}$ & 11 & 4788 & Observational & $\begin{array}{l}\text { Serious } \\
(-1)\end{array}$ & $\begin{array}{l}\text { Consistency (+ } \\
\text { 1) }\end{array}$ & Not Serious & NO & NO & Low & Critical \\
\hline \multicolumn{11}{|c|}{ Short Physical Performance Battery (SPPB) } \\
\hline $\begin{array}{l}\text { All-Cause } \\
\text { Mortality }\end{array}$ & 2 & 243 & Observational & $\begin{array}{l}\text { Very } \\
\text { Serious } \\
(-2)\end{array}$ & Serious $(-1)$ & Not Serious & Serious $(-1)$ & NO & Very Low & Critical \\
\hline $\begin{array}{l}\text { Hospitalisation } \\
\text { and Mortality }\end{array}$ & 3 & 231 & Observational & $\begin{array}{l}\text { Serious } \\
(-1)\end{array}$ & Not Serious & Not Serious & Not Serious & NO & Very Low & Critical \\
\hline \multicolumn{11}{|l|}{ Gait Speed } \\
\hline $\begin{array}{l}\text { All-Cause } \\
\text { Mortality }\end{array}$ & 7 & 4828 & Observational & NO & Not Serious & NO & Not Serious & NO & Low & Critical \\
\hline $\begin{array}{l}\text { All-Cause } \\
\text { Hospitalisation }\end{array}$ & 4 & 2002 & Observational & NO & Not Serious & NO & Not Serious & NO & Low & Critical \\
\hline $\begin{array}{l}\text { HF } \\
\text { Hospitalisation }\end{array}$ & 2 & 719 & Observational & NO & Not Serious & NO & Not Serious & NO & Low & Critical \\
\hline $\begin{array}{l}\text { Hospitalisation } \\
\text { and Mortality }\end{array}$ & 2 & 1146 & Observational & NO & Not Serious & NO & Not Serious & NO & Low & Critical \\
\hline
\end{tabular}

In brief, the GRADE classification was carried out according to the presence, or not, of the following identified factors: (1) study design, (2) risk of bias, (3) inconsistency of results (4) indirectness (5) imprecision, and (6) other considerations (e.g. reporting bias). The quality of the evidence based on the GRADE criteria was classified as: (1) high (further research is unlikely to change our confidence in the estimate of effect and there are no known or suspected reporting bias); (2) moderate (further research is likely to have an important effect on our confidence in the estimate of effect and could change the estimate); ( 3 ) low (further research is likely to have an important effect on our confidence in the estimate of effect and is likely to change the estimate); or (4) very low (we are uncertain about the estimate) [38]

a Design: Observational Longitudinal Cohort Studies show a Low Level of Evidence according to GRADE

${ }^{b}$ Risk Of Bias: $>50 \%$ (NO) of the information is from studies with low risk of bias which rarely can affect the interpretation of results. $50 \%$ (Not Serious) of the information is from studies with moderate risk of bias which could affect the interpretation of results, and $50 \%$ of the information is from studies with low risk of bias. $>50 \%$ (Serious) or $>75 \%$ (Very Serious) of the information is from studies with high/moderate risk of bias which sufficiently can affect the interpretation of results

c Inconsistency: $>50 \%$ (Consistency) presence of high degree of consistency in the results, such as effects in same directions and not variations in the degree to which the outcome is affected (large significant effects (Hazard Ratio or Odds Ratio > 2)). > 50\% (Not Serious) presence of high degree of consistency in the results, such as effects in same directions although variations in the degree to which the outcome is affected (small significant effects or large significant effects). $>50 \%$ (Serious) or $>75 \%$ (Very serious) presence of high degree of inconsistency in the results, such as effects in opposite directions, or large variations in the degree to which the outcome is affected (eg, very large and very small effects or no significant effect)

${ }^{d}$ Indirectness: $>50 \%$ (NO) of included studies report similar population (similar HF diagnosis and similar age), as well as the same functional test (although different distances or cut-off points) and the same outcome. $>50 \%$ (Not Serious) of included studies show different HF diagnosis but population with similar age, and the same functional test (although different distances or cut-off points) and the same outcome is reported

e Imprecision: $>50 \%$ (NO) of included studies report a $95 \% \mathrm{Cl}$, with a narrow range (it excludes 1.0), includes large effects in the same direction and the sample size is large. $>50 \%$ (Not Serious) of included studies report a $95 \% \mathrm{Cl}$, with a narrow range (it excludes 1.0 ), includes large or small effects in the same direction and the sample size could be small. $>50 \%$ (Serious) or $>75 \%$ (Very Serious) of included studies present $95 \%$ Cls with wide range (it does not exclude 1.0 ) and includes small effects in both directions

f Other: Publication Bias is not suspected, and $>75 \%$ of included studies included the outcome data in a multivariate models adjusted by variables which could change the effect (NO)

increased risk of the combined endpoint of hospitalisation and mortality for any cause [84], specially when gait speed was slower than $0.80 \mathrm{~m} / \mathrm{s}[83,84,86]$. GRADE criteria reported a low level of evidence per outcome in each prognostic outcome in Gait Speed Test. Other studies have shown the relationship between gait speed and survival, death and hospitalisation due to HF [27, 94]. In fact, Dodson et al. [95] revealed that patients who showed a gait speed slower than $0.8 \mathrm{~m} / \mathrm{s}$ were more likely to experience one-year mortality or hospitalisation than patients with gait speed faster than $0.8 \mathrm{~m} / \mathrm{s}$. Alfredsson et al. [96] also reported that patients with a 
gait speed slower than $0.8 \mathrm{~m} / \mathrm{s}$ after a transcatheter aortic valve replacement, had 35\% higher 30 -day mortality than patients with faster gait speed. Chainani et al. [97] reported that gait speed and handgrip strength are associated with increased risk of cardiovascular mortality.

A meta-analysis published by Yamamoto et al. [98] reported that 6MWT were significantly associated with mortality and cardiovascular disease. Frailty has also been associated with larger risk of mortality and hospitalisation in patients with chronic $\operatorname{HF}[25,26,30,31$, 99]. Bagnall et al. [100] revealed that frailty patients had a risk of mortality 2- to 4-fold compared with non-frail patients after acardiac surgery or transcatheter aortic valve implantation. Gait speed is a marker of frailty, although frailty could be also assessed by the 6MWT, the SPPB or the TUG [25, 26, 30, 31, 99]. In this way, the use of functional tests seem to be useful to stratify patients with HF based on their physical functional performance and to determine their prognosis.

To our knowledge, our review is the first systematic review reporting the level of evidence per each prognostic outcome using GRADE criteria. Other reviews showed the prognostic role of the 6MWT test or the impact of the physical performance on prognosis in patients with HF, but not reported the risk of bias of included studies or the level of evidence per outcome according to GRADE criteria [22, 23, 98, 101-103].

\section{Implications for clinical practice}

The current findings may be useful to promote functional assessments that allow stratify patients with HF according to their functional impairment. Furthermore, accurate prognostic stratification could be essential for optimizing clinical management and treatment decision making, with the aim of maintaining functionality, improving quality of life and reducing the number of hospitalisations, as well as increasing the life expectancy of patients with HF.

Adjusted medical-pharmacological treatment, in addition to improve symptoms, could prevent further cardiovascular accidents and prolong the life expectancy of patients with HF [13]. Moreover, adjusted exercise programs could reduce mortality, may improve functional capacity and quality of life, and may reduce hospitalisations $[5,8]$. It has also been shown that patients with more physical activity performed weekly reported a lower risk of mortality [104-106]. Functional tests such as 6MWT, Gait Speed or SPPB may provide incremental prognostic value and could help to individualize the exercise prescription [107].

\section{Future research}

Future research should aim to determine the optimal cut-off points for prognostic prediction and to determine the utility of functional assessments in the management and treatment of patients with HF. The following recommendations should guide future research: 1) use the same cut off point in functional tests; 2) include a large sample size with patients with HF who show different characteristics.

\section{Strengths and limitations of the study}

The strengths of this systematic review and metaanalysis included the use of a pre-specified protocol registered on PROSPERO, the PRISMA checklist, the NOS to determine the risk of bias of each study, the GRADE criteria to assess the overall quality and the strength of the evidence per outcome, a robust search strategy complemented by a manual search, so that all studies that met the eligibility criteria could have been identified. Thus, our systematic review included 44 studies, while a previous similar review carried out by Yamamoto et al. [98] included only 22 studies.

However, there are several limitations that should be mentioned. First, the lack of uniformity among included studies, which included different cut-off points in functional tests, should be taken into account when interpreting the results. Finally, most of prognositc outcomes showed a low level of evidence per outcome according to GRADE criteria.

\section{Conclusion}

Patients with HF who report a poor physical functional performance in the 6MWT, in the SPPB or in the Gait Speed Test, show worse prognosis than patients who report a good physical functional performance in terms of an increased risk of hospitalisation or an increased risk of mortality. However, there is a lack of homogeneity regarding which cut-off point should be used to stratify patients with HF based on their physical functional performance in the different functional tests and GRADE criteria show a low level of evidence per outcome in most of examined prognostic outcome variables.

\section{Supplementary information}

Supplementary information accompanies this paper at https://doi.org/10. 1186/s12872-020-01725-5.

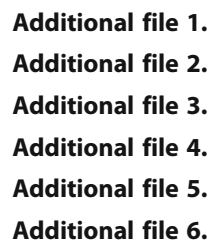

HF: Heart failure; 6MWT: Six minute walking test; SPPB: Short physical performance battery; DALYs: Disability-adjusted life-years; TUG: Timed up and go test; PECOS: Participant, exposure, comparator, outcome, study design; 
PRISMA: Preferred reporting items for systematic reviews and meta-analyses statement; PROSPERO: International prospective register of systematic reviews; NYHA: New York heart association; OR: Odds ratio; HR: Hazard ratio NOS: The Newcastle Ottawa scale; GRADE: Grading of recommendations assessment, development and evaluation; HFrEF: Heart failure with reduced ejection fraction; HFpEF: Heart failure with preserved ejection fraction; $\mathrm{Cl}$ : Confidence interval; m: Meters

\section{Acknowledgments}

Not Applicable.

\section{Authors' contributions}

IJF-A and AC-V contributed to the conception of this study. IJF-A and AC-V were involved in the selection and analysis of the included studies. IJF-A, AC$V, B-S, L M P-B, M R B-L$ and $R G-H$ were involved in the writing and in the review of the manuscript. All authors read and approved the manuscript.

\section{Funding}

Brendon Stubbs is supported by a Clinical Lectureship (ICA-CL-2017-03-001) jointly funded by Health Education England (HEE) and the National Institute for Health Research (NIHR). Brendon Stubbs is part funded by the NIHR Biomedical Research Centre at South London and Maudsley NHS Foundation Trust. Brendon Stubbs is also supported by the Maudsley Charity, King's College London and the NIHR South London Collaboration for Leadership in Applied Health Research and Care (CLAHRC) funding. This paper presents independent research. The views expressed in this publication are those of the authors and not necessarily those of the acknowledged institutions. María Rosa Bernal-López was supported by "Miguel Servet Type I" program (CP15/00028) from the ISCIII-Madrid (Spain), cofinanced by the Fondo Europeo de Desarrollo Regional-FEDER.

\section{Availability of data and materials}

Not Applicable.

\section{Ethics approval and consent to participate}

Not Applicable.

\section{Consent for publication}

Not Applicable.

\section{Competing interests}

The authors declare that they have no conflict of interest.

\begin{abstract}
Author details
${ }^{1}$ Department of Physiotherapy, Faculty of Health Science, University of Malaga, The Institute of Biomedical Research in Malaga (IBIMA), Clinimetric Group FE-14, Malaga, Spain. ²Physiotherapy Department, South London and Maudsley NHS Foundation Trust, Denmark Hill, London, UK. ${ }^{3}$ Department of Psychological Medicine, Institute of Psychiatry, Psychology and Neuroscience,

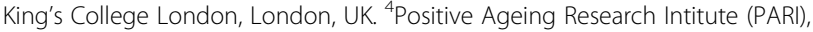
Faculty of Health Social Care and Education, Anglia Ruskin University, Chelmsford, UK. ${ }^{5}$ Internal Medicine Department, Instituto de Investigación Biomédica de Malaga (IBIMA), Regional University Hospital of Málaga, Málaga, Spain. ${ }^{6}$ Unidad de Neurofisiología Cognitiva, Centro de Investigaciones Médico Sanitarias (CIMES), Instituto de Investigación Biomédica de Málaga (IBIMA), Universidad de Málaga (UMA), Campus de Excelencia Internacional (CEI) Andalucía Tech, Málaga, Spain. ${ }^{7}$ Centro de Investigación Biomédica en Red Enfermedades Cardiovasculares (CIBERCV), Instituto de Salud Carlos III, Madrid, Spain. ${ }^{8}$ CIBER Fisio-patología de la Obesidad y la Nutrición, Instituto de Salud Carlos III, Madrid, Spain. ${ }^{9}$ School of Clinical Sciences, Faculty of Health at the Queensland University of Technology, Brisbane, Queensland, Australia.
\end{abstract}

Received: 23 July 2020 Accepted: 4 October 2020

Published online: 09 December 2020

\section{References}

1. Abajobir AA, Abate KH, Abbafati C, Abbas KM, Abd-Allah F, Abdulkader RS, et al. Global, regional, and national disability-adjusted life-years (DALYs) for 333 diseases and injuries and healthy life expectancy (HALE) for 195 countries and territories, 1990-2016: a systematic analysis for the global burden of disease study 2016. Lancet. 2017;390(10100):1260-344.

2. Abajobir AA, Abbafati C, Abbas KM, Abd-Allah F, Abera SF, Aboyans V, et al. Global, regional, and national age-sex specific mortality for 264 causes of death, 1980-2016: a systematic analysis for the global burden of disease study 2016. Lancet. 2017;390(10100):1151-210.

3. Wang H, Abajobir AA, Abate KH, Abbafati C, Abbas KM, Abd-Allah F, et al. Global, regional, and national under-5 mortality, adult mortality, age-specific mortality, and life expectancy, 1970-2016: a systematic analysis for the global burden of disease study 2016. Lancet. 2017;390(10100):1084-150.

4. Bui A, Horwich T, Fonarow G. Epidemiology and risk profile of heart failure. Nat Rev Cardiol. 2011;8(3):30-41.

5. Rogers C, Bush N. Heart failure: pathophysiology, diagnosis, medical treatment guidelines, and nursing management. Nurs Clin North Am. 2015;50(4): 787799. DOl: http://dx.doi.org/https://doi.org/10.1016/j.cnur.2015.07.012.

6. Tendera M. Epidemiology, treatment, and guidelines for the treatment of heart failure in Europe. Eur Heart J. 2005;7(1):5-9.

7. Smith AC. Effect of telemonitoring on re-admission in patients with congestive heart failure. Medsurg Nurs. 2013;22(1):39-44.

8. Yancy $C$, Jessup M, Bozkurt B, et al. ACCF/AHA guideline for the management of heart failure: a report of the American College of Cardiology Foundation/American Heart Association task force on practice guidelines. J Am Coll Cardiol. 2013;62(16):147.

9. Wheeler EC, Plowfield L. Clinical education initiative in the community: caring for patients with congestive heart failure. Nurs Educ Perspect. 2004; 25(1):16-21.

10. Fletcher $L$, Thomas D. Heart failure: understanding the pathophysiology and management. J Am Acad Nurse Pract. 2001;13(6):249-57.

11. Siracuse J, Chaikof E. The pathogenesis of diabetic atherosclerosis. Diab Peripher Vasc Dis Diagn Manage. 2012;158(5):13-26.

12. Kaminsky LA, Tuttle MS. Functional assessment of heart failure patients. Heart Fail Clin. 2015;11(1):29-36. doi: http://dx.doi.org/https://doi.org/10. 1016/j.hfc.2014.08.002.

13. Ponikowski P, Voors AA, Anker SD, et al. ESC guidelines for the diagnosis and treatment of acute and chronic heart failure: the task forcé for the diagnosis and treatment of acute and chronic heart failure of the European society of cardiology (ESC) developed with the special contribution of the heart failure association (HFA) of the ESC. Eur Heart J. 2016;37:2129-200.

14. Gutekunst DJ. Isokinetic torque timing parameters and ceramides as markers of muscle dysfunction in systolic heart failure. J Card Fail. 2016;22(5): 356-357. doi: http://dx.doi.org/https://doi.org/10.1016/j.cardfail.2016.03.018.

15. Watson RD, Gibbs CR, Lip GY. ABC of heart failure. Clinical features and complications. BMJ. 2000;320(7229):236-9.

16. Barker J, Byrne KS, Doherty A, Foster C, Rahimi K, Ramakrishnan R, et al. Physical activity of UK adults with chronic disease: cross-sectional analysis of accelerometer-measured physical activity in 96706 UK biobank participants. Int J Epidemiol. 2019;48(4):1167-74.

17. Kinugawa S, Takada S, Matsushima S, Okita K, Tsutsui H. Skeletal muscle abnormalities in heart failure. Int Heart J. 2015;56(5):475-84.

18. Bona RL, Bonezi A, da Silva PF, Biancardi CM, de Souza Castro FA, Clausel NO. Effect of walking speed in heart failure patients and heart transplant patients. Clin Biomech. 2017;42: 85-91. doi: http://dx.doi.org/https://doi.org/ 10.1016/j.clinbiomech.2017.01.008.

19. Mancini DM, Eisen H, Kussmaul W, Mull R, Edmunds L, Wilson J. Value of peak exercise oxygen consumption for optimal timing of cardiac transplantation in ambulatory patients with heart failure. Circulation. 1991;83(3):778-86.

20. Myers J, Prakash M, Froelicher V, Do D, Partington S, Atwood JE. Exercise capacity and mortality among men referred for exercise testing. N Engl J Med. 2002;346(11):793-801.

21. Hülsmann M, Quittan $M$, Berger R, Crevenna R, Springer C, Nuhr M, et al. Muscle strength as a predictor of long-term survival in severe congestive heart failure. Eur J Heart Fail. 2004;6(1):101-7.

22. Rostagno C, Gensini GF. Six minute walk test: a simple and useful test to evaluate functional capacity in patients with heart failure. Intern Emerg Med. 2008;3(3):205-12.

23. Du H, Wonggom P, Tongpeth J, Clark RA. Six-minute walk test for assessing physical functional capacity in chronic heart failure. Curr Heart Fail Rep. 2017;14(3):158-66.

24. Rasekaba T, Lee AL, Naughton MT, Williams TJ, Holland AE. The six-minute walk test: A useful metric for the cardiopulmonary patient. Intern Med J. 2009;39:495-501. 
25. Díez-Villanueva P, Arizá-Solé A, Vidán MT, Bonanad C, Formiga F, Sanchis J, et al. Recomendaciones de la Sección de Cardiología Geriátrica de la Sociedad Española de Cardiología Para la valoración de la fragilidad en el anciano con cardiopatía. Rev Esp Cardiol. 2019;72(1):63-71.

26. Yang X, Lupón J, Vidán MT, Ferguson C, Gastelurrutia P, Newton PJ, et al. Impact of frailty on mortality and hospitalisation in chronic heart failure: a systematic review and meta-analysis. J Am Heart Assoc. 2018;7(23):e008251.

27. Studenski S, Perera S, Kushang P, Rosano C, Faulkner K, Inzitari M, et al. Gait speed and survival in older adults. JAMA. 2011;305(1):50-8.

28. Abellan van Kan G, Rolland Y, Andrieu S, et al. Gait speed at usual pace as a predictor of adverse outcomes in community-dwelling older people an international academy on nutrition and aging (IANA) task force. J Nutr Health Aging. 2009;13:881-9.

29. Afilalo J, Alexander KP, Mack MJ, et al. Frailty assessment in the cardiovascular care of older adults. J Am Coll Cardiol. 2014;63:747-62.

30. Singh M, Stewart $R$, White $H$. Importance of frailty in patients with cardiovascular disease. Eur Heart J. 2014;35:1726-31.

31. Newman AB, Gottdiener JS, McBurnie MA, et al. Associations of subclinical cardiovascular disease with frailty. J Gerontol A Biol Sci Med Sci. 2001;56: M158-66.

32. Guyatt GH, Sullivan MJ, Thompson PJ, Fallen EL. Pugsley S0, Taylor DW, et al. the 6-minute walk: a new measure of exercise capacity in patients with chronic heart failure. Can Med Assoc J. 1985;132(8):919-23.

33. Guralnik JM, Simonsick EM, Ferrucci L, Glynn RJ, Berkman LF, Blazer DG, et al. A short physical performance battery assessing lower extremity function: association with self-reported disability and prediction of mortality and nursing home admission. J Gerontol. 1994;49(2):85-94.

34. Podsiadlo D, Richardson S. The timed "up and go": a test of basic functional mobility for frail elderly persons. J Am Geriatr Soc. 1991;39(2):142-8.

35. Chan ME, Arvey RD. Meta-analysis and the development of knowledge. Perspect Psychol Sci. 2012;7:79-92. https://doi.org/10.1177/ 1745691611429355.

36. Liberati A, Altman DG, Tetzlaff J, Mulrow C, Gotzsche PC, loannidis JP, Clarke M, Devereaux PJ, Kleijnen J, Moher D. The PRISMA statement for reporting systematic reviews and meta-analyses of studies that evaluate healthcare interventions: explanation and elaboration. BMJ. 2009;339:b2700.

37. Haddaway NR, Collins AM, Coughlin D, et al. The role of google scholar in evidence reviews and its applicability to grey literature searching. PLoS One. 2015;10:e0138237-17. https://doi.org/10.1371/journal.pone.0138237.

38. Wells GA, Shea B, O'Connell D, Peterson J, Welch V, Losos M, et al. The Newcastle-Ottawa Scale (NOS) for assessing the quality of non-randomised studies in meta-analyses. 2008 [Accessed September 24, 2019]. Available from URL: http://www.ohri.ca/programs/clinical_epidemiology/oxford.asp.

39. Zeng X, Zhang Y, Kwong JSW, Zhang C, Li S, Sun F, et al. The methodological quality assessment tools for preclinical and clinical studies, systematic review and meta-analysis, and clinical practice guideline: a systematic review. J Evid Based Med. 2015;8:2-10. https://doi.org/10.1111/ jebm.12141.

40. Atkins $D$, Best $D$, Briss PA, et al. Grading quality of evidence and strength of recommendations. BMJ. 2004;328:1490. https://doi.org/10.1136/bmj.328. 7454.1490 .

41. Guyatt GH, Oxman AD, Vist GE, Kunz R, Falck-Ytter Y, Alonso-Coello P, Schünemann HJ. GRADE working group: GRADE: an emerging consensus on rating quality of evidence and strength of recommendations. BMJ. 2008; 336:924-6. https://doi.org/10.1136/bmj.39489.470347.AD.

42. Higgins JP, Thompson SG, Deeks JJ, Altman DG. Measuring inconsistency in meta-analyses. BMJ. 2003:327:557-60.

43. Higgins JP, Thompson SG. Quantifying heterogeneity in a meta-analysis. Stat Med. 2002;21:1539-58.

44. Review Manager (RevMan) [Computer program]. Version 5.3. Copenhagen: The Nordic Cochrane Centre, The Cochrane Collaboration, 2014.

45. Brenyo A, Goldenberg I, Moss AJ, Rao M, McNitt S, Huang DT, et al. Baseline functional capacity and the benefit of cardiac resynchronization therapy in patients with mildly symptomatic heart failure enrolled in MADIT-CRT. Hear Rhythm 2012;9(9):1454-1459. Available from: http://dx.doi.org/https://doi. org/10.1016/j.hrthm.2012.04.018.

46. Ferreira JP, Metra M, Anker SD, Dickstein K, Lang CC, Ng L, et al. Clinical correlates and outcome associated with changes in 6-minute walking distance in patients with heart failure: findings from the BIOSTAT-CHF study. Eur J Heart Fail. 2019;21(2):218-26.
47. Wegrzynowska-Teodorczyk K, Rudzinska E, Lazorczyk M, Nowakowska K, Banasiak W, Ponikowski P, et al. Distance covered during a six-minute walk test predicts long-term cardiovascular mortality and hospitalisation rates in men with systolic heart failure: an observational study. J Physiother. 2013; 59(3):177-87.

48. Bittner V, Weiner DH, Yusuf S, Rogers WJ, Mcintyre KM, Bangdiwala SI, et al. Prediction of mortality and morbidity with a 6-minute walk test in patients with left ventricular dysfunction. JAMA. 1993;270(14):1702-7.

49. Arslan S, Erol MK, Gundogdu F, Sevimli S, Aksakal E, Senocak H, et al. Prognostic value of 6-minute walk test in stable outpatients with heart failure. Tex Heart Inst J. 2007;34(2):166-9.

50. Lee R, Chan YH, Wong J, Lau D, Ng K. The 6-minute walk test predicts clinical outcome in Asian patients with chronic congestive heart failure on contemporary medical therapy: a study of the multiracial population in Singapore. Int J Cardiol. 2007 Jul 10;119(2):168-75.

51. Curtis JP, Rathore SS, Wang Y, Krumholz HM. The association of 6-minute walk performance and outcomes in stable outpatients with heart failure. Card Fail. 2004;10(1):9-14.

52. Ingle L, Cleland JG, Clark AL. The long-term prognostic significance of 6minute walk test distance in patients with chronic heart failure. Biomed Res Int. 2014:2014:505969.

53. Alahdab MT, Mansour IN, Napan S, Stamos TD. Six minute walk test predicts long-term all-cause mortality and heart failure Rehospitalisation in AfricanAmerican patients hospitalized with acute decompensated heart failure. J Card Fail 2009;15(2): 130-135. Available from: http://dx.doi.org/https://doi. org/10.1016/j.cardfail.2008.10.006.

54. Mangla A, Kane J, Beaty E, Richardson D, Powell LH, Calvin JE. Comparison of predictors of heart failure-related hospitalisation or death in patients with versus without preserved left ventricular ejection fraction. Am J Cardiol. 2013;112(12):1907-12

55. Hasin T, Topilsky Y, Kremers WK, Boilson BA, Schirger JA, Edwards BS, et al. Usefulness of the six-minute walk test after continuous axial flow left ventricular device implantation to predict survival. Am J Cardiol 2012;110(9): 1322-1328. Available from: http://dx.doi.org/https://doi.org/10.1016/j. amjcard.2012.06.036.

56. Passantino A, Lagioia R, Mastropasqua F, Scrutinio D. Short-term change in distance walked in $6 \mathrm{~min}$ is an Indicator of outcome in patients with chronic heart failure in clinical practice. J Am Coll Cardiol. 2006;48(1):99-105.

57. Howie-Esquivel J, Dracup K. Does oxygen saturation or distance walked predict rehospitalisation in heart failure? J Cardiovasc Nurs. 2008 Jul;23(4): 349-56.

58. Zotter-Tufaro C, Mascherbauer J, Duca F, Koell B, Aschauer S, Kammerlander $A A$, et al. Prognostic significance and Determinantsof the 6-min walk test inPatients WithHeart failure and preserved EjectionFraction. JACC Hear Fail. 2015:3(6):459-66.

59. Boxer R, Kleppinger A, Ahmad A, Annis K, Hager D, Kenny A. The 6-minute walk is associated with frailty and predicts mortality in older adults with heart failure. Congest Hear Fail. 2010;16(5):208-13.

60. Ingle L, Cleland JG, Clark AL. The relation between repeated 6-minute walk test performance and outcome in patients with chronic heart failure. Ann Phys Rehabil Med. 2014;57(4):244-53.

61. Guazzi M, Dickstein K, Vicenzi M, Arena R. Six-minute walk test and cardiopulmonary exercise testing in patients with chronic heart failure: a comparative analysis on clinical and prognostic insights. Circ Hear Fail. 2009; 2(6):549-55.

62. McCabe N, Butler J, Dunbar SB, Higgins M, Reilly C. Six-minute walk distance predicts 30-day readmission after acute heart failure hospitalisation. Heart Lung. 2017;46(4):287-92.

63. Vegh EM, Kandala J, Orencole M, Upadhyay GA, Sharma A, Miller A, et al. Device-measured physical activity versus six-minute walk test as a predictor of reverse remodeling and outcome after cardiac resynchronization therapy for heart failure. Am J Cardiol. 2014;113(9):1523-8.

64. Roul G, Germain P, Bareiss P. Does the 6-minute walk test predict the prognosis in patients with NYHA class II or III chronic heart failure? Am Heart J. 1998;136(3):449-57.

65. Frankenstein L, Remppis A, Graham J, Schellberg D, Sigg C, Nelles M, et al. Gender and age related predictive value of walk test in heart failure: Do anthropometrics matter in clinical practice? Int J Cardiol. 2008;127(3):331-6.

66. Mene-Afejuku TO, Balogun MO, Akintomide AO, Adebayo RA. Prognostic indices among hypertensive heart failure patients in Nigeria: the roles of $24-$ 
hour holter electrocardiography and 6-minute walk test. Vasc Health Risk Manag. 2017;13:71-9.

67. Ingle L, Rigby AS, Carroll S, Butterly R, King RF, Cooke CB, et al. Prognostic value of the $6 \mathrm{~min}$ walk test and self-perceived symptom severity in older patients with chronic heart failure. Eur Heart J. 2007;28:560-8.

68. Rostagno C, Olivo G, Comeglio M, Boddi V, Banchelli M, Galanti G, et al. Prognostic value of 6-minute walk corridor test in patients with mild to moderate heart failure: comparison with other methods of functional evaluation. Eur J Heart Fail. 2003;5(3):247-52.

69. Cahalin LP, Mathier MA, Semigran MJ, Dec GW, DiSalvo TG. The six-minute walk test predicts peak oxygen uptake and survival in patients with advanced heart failure. Chest. 1996;110(2):325-32.

70. Frankenstein L, Zugck C, Nelles M, Schellberg D, Katus H, Remppis A. Sexspecific predictive power of 6-minute walk test in chronic heart failure is not enhanced using percent achieved of published reference equations. J Hear Lung Transplant. 2008;27(4):427-34.

71. Rubim VSM, Neto CD, Martins-Romeo JL, Montera MW. Valor prognóstico do teste de caminhada de seis minutos na insuficiência cardíaca. Arq Bras Cardiol. 2006;86(2):120-5.

72. Kanagala P, Arnold JR, Cheng ASH, Singh A, Khan JN, Gulsin GS, et al. Left atrial ejection fraction and outcomes in heart failure with preserved ejection fraction. Int J Cardiovasc Imaging. 2020 Jan 1;36(1):101-10.

73. Zugck C, Krüger C, Kell R, Körber S, Schellberg D, Kübler W, et al. Risk stratification in middle-aged patients with congestive heart failure: prospective comparison of the heart failure survival score (HFSS) and a simplified two-variable model. Eur J Heart Fail. 2001;3(5):577-85.

74. Cahalin LP, Arena R, Labate V, Bandera F, Lavie CJ, Guazzi M. Heart rate recovery after the 6 min walk test rather than distance ambulated is a powerful prognostic Indicator in heart failure with reduced and preserved ejection fraction: a comparison with cardiopulmonary exercise testing. Eur J Heart Fail. 2013;15:519-27.

75. Reibis RK, Treszl A, Wegscheider K, Ehrlich B, Dissmann R, Völler H. Exercise capacity is the most powerful predictor of 2-year mortality in patients with left ventricular systolic dysfunction. Herz. 2010 Mar;35(2):104-10.

76. Castel MA, Méndez F, Tamborero D, Mont L, Magnani S, Tolosana JM, et al. Six-minute walking test predicts long-term cardiac death in patients who received cardiac resynchronization therapy. Europace. 2009;11:338-42.

77. Kamiya K, Hamazaki N, Matsue Y, Mezzani A, Corrà U, Matsuzawa R, et al. Gait speed has comparable prognostic capability to six-minute walk distance in older patients with cardiovascular disease. Eur J Prev Cardiol. 2018:25(2):212-9.

78. García GL, Sánchez SM, García-Briñón MÁ, Fernández-Alonso C, González del Castillo J, Martín-Sánchez FJ. El efecto de la fragilidad física en el pronóstico a largo plazo en los pacientes mayores con insuficiencia cardiaca aguda dados de Alta desde un servicio de urgencias. Emergencias. 2019;31(6):413-6.

79. Hornsby WE, Sareini M, Golbus JR, Willer J, Mcnamara JL, Konerman MC, et al. Lower extremity function is independently associated with hospitalisation burden in heart failure with preserved ejection fraction. J Card Fail. 2019;25(1):2-9.

80. Chiarantini D, Volpato S, Sioulis F, Bartalucci F, Del Bianco L, Mangani I, et al. Lower extremity performance measures predict long-term prognosis in older patients hospitalized for heart failure. J Card Fail. 2010;16(5):390-5.

81. Zaharias E, Cataldo J, Mackin L, Howie-Esquivel J. Simple measures of function and symptoms in hospitalized heart failure patients predict shortterm cardiac event-free survival. Nurs Res Pract. 2014;815984.

82. Lo AX, Donnelly JP, McGwin G, Bittner V, Ahmed A, Brown CJ. Impact of gait speed and instrumental activities of daily living on all-cause mortality in adults $\geq 65$ years with heart failure. Am J Cardiol. 2015;115(6):797-801.

83. Pulignano G, Del Sindaco D, Di Lenarda A, Alunni G, Senni M, Tarantini L, et al. Incremental value of gait speed in predicting prognosis of older adults with heart failure: insights from the IMAGE-HF study. JACC Hear Fail. 2016; 4(4):289-98.

84. Chaudhry SI, McAvay G, Chen S, Whitson H, Newman AB, Krumholz HM, et al. Risk factors for hospital admission among older persons with newly diagnosed heart failure: findings from the cardiovascular health study. J Am Coll Cardiol. 2013;61 (6):635-42

85. Tanaka S, Kamiya K, Hamazaki N, Matsuzawa R, Nozaki K, Maekawa E, et al. Incremental value of objective frailty assessment to predict mortality in elderly patients hospitalized for heart failure. J Card Fail. 2018;24(11):723-32.
86. Tanaka S, Kamiya K, Hamazaki N, Matsuzawa R, Nozaki K, Nakamura T, et al. Short-term change in gait speed and clinical outcomes in older patients with acute heart failure. Circ J. 2019;83(9):1860-7.

87. Rodríguez-Pascual C, Paredes-Galán E, Ferrero-Martínez Al, GonzálezGuerrero JL, Hornillos-Calvo M, Menendez-Colino R, et al. The frailty syndrome is associated with adverse health outcomes in very old patients with stable heart failure: a prospective study in six Spanish hospitals. Int J Cardiol. 2017;236:296-303.

88. Vidán MT, Blaya-Novakova V, Sánchez E, Ortiz J, Serra-Rexach JA, Bueno H. Prevalence and prognostic impact of frailty and its components in nondependent elderly patients with heart failure. Eur J Heart Fail. 2016;18(7): $869-75$.

89. Shoemaker MJ, Curtis AB, Vangsnes E, Dickinson MG. Clinically meaningful change estimates for the six-minute walk test and daily activity in individuals with chronic heart failure. Cardiopulm Phys Ther J. 2013;24(3): 21-9.

90. Fox KR, Ku P-W, Hillsdon M, Davis MG, Simmonds BAJ, Thompson JL, et al. Objectively assessed physical activity and lower limb function and prospective associations with mortality and newly diagnosed disease in UK older adults: an OPAL four-year follow-up study. Age Ageing. 2015;44(2): 261-8.

91. Corsonello A, Lattanzio F, Pedone C, Garasto S, Laino I, Bustacchini S, et al. Prognostic significance of the short physical performance battery in older patients discharged from acute care hospitals. Rejuvenation Res. 2012;15(1): $41-8$.

92. Legrand D, Vaes B, Matheï C, Adriaensen W, Van Pottelbergh G, Degryse JM. Muscle strength and physical performance as predictors of mortality, hospitalisation, and disability in the oldest old. J Am Geriatr Soc. 2014;62(6): 1030-8.

93. Volpato S, Cavalieri M, Sioulis F, Guerra G, Maraldi C, Zuliani G, et al. Predictive value of the short physical performance battery following hospitalisation in older patients. J Gerontol A Biol Sci Med Sci. 2011;66(1): 89-96.

94. Reeves GR, Forman DE. Gait speed: stepping towards improved assessment of heart failure patients. JACC Hear Fail. 2016;4(4): 299-300. doi: http://dx. doi.org/https://doi.org/10.1016/j.jchf.2016.02.002.

95. Dodson JA, Arnold SV, Gosch KL, Gill TM, Spertus J, Krumholz HM, et al. Slow gait speed and risk of mortality or hospital readmission following myocardial infarction in the TRIUMPH registry. J Am Geriatr Soc. 2016;64(3): 596-601.

96. Alfredsson J, Stebbins A, Brennan JM, Matsouaka R, Afilalo J, Peterson ED, et al. Gait speed predicts 30-day mortality after Transcatheter aortic valve replacement: results from the Society of Thoracic Surgeons/American College of Cardiology Transcatheter Valve Therapy Registry. Circulation. 2016;133(14):1351-9.

97. Chainani V, Shaharyar S, Dave K, Choksi V, Ravindranathan S, Hanno R, et al. Objective measures of the frailty syndrome (hand grip strength and gait speed) and cardiovascular mortality: a systematic review. Int I Cardiol. 2016; 215:487-93.

98. Yamamoto S, Yamaga T, Nishie K, Sakai Y, Ishida T, Oka K, et al. Impact of physical performance on prognosis among patients with heart failure: systematic review and meta-analysis. J Cardiol. 2020;76(2):139-46.

99. Boxer RS, Wang Z, Walsh SJ, Hager D, Kenny AM. The utility of the 6-minute walk test as a measure of frailty in older adults with heart failure. Am J Geriatr Cardiol. 2008;17(1):7-12.

100. Bagnall NM, Faiz O, Darzi A, Athanasiou T. What is the utility of preoperative frailty assessment for risk stratification in cardiac surgery? Interact Cardiovasc Thorac Surg. 2013;17(2):398-402.

101. Giannitsi S, Bougiakli M, Bechlioulis A, Kotsia A, Michalis LK, Naka KK. 6minute walking test: a useful tool in the management of heart failure patients. Ther Adv Cardiovasc Dis. 2019;13:1-10.

102. Zielinska D, Bellwon J, Rynkiewicz A, Elkady MA. Prognostic value of the sixminute walk test in heart failure patients undergoing cardiac surgery: a literature review. Rehabil Res Pract. 2013;2013:965494.

103. Pollentier B, Irons SL, Benedetto CM, Dibenedetto A-M, Loton D, Seyler RD, et al. Examination of the six minute walk test to determine functional capacity in people with chronic heart failure: a systematic review. Cardiopulm Phys Ther J. 2010;21(1):13-21.

104. Jeong SW, Kim SH, Kang SH, Kim HJ, Yoon CH, Youn TJ, et al. Mortality reduction with physical activity in patients with and without cardiovascular disease. Eur Heart J. 2019;40(43):3547-55. 
105. Kraus WE, Powell KE, Haskell WL, Janz KF, Campbell WW, Jakicic JM, et al. Physical activity, all-cause and cardiovascular mortality, and cardiovascular disease. Med Sci Sports Exerc. 2019;51(6):1270-81.

106. Blond K, Brinkløv CF, Ried-Larsen M, Crippa A, Grøntved A. Association of high amounts of physical activity with mortality risk: a systematic review and meta-analysis. Br J Sports Med. 2019:1-8.

107. Afilalo J. Evaluating and treating frailty in cardiac rehabilitation. Clin Geriatr Med. 2019;35(4):445-57.

\section{Publisher's Note}

Springer Nature remains neutral with regard to jurisdictional claims in published maps and institutional affiliations.

Ready to submit your research? Choose BMC and benefit from:

- fast, convenient online submission

- thorough peer review by experienced researchers in your field

- rapid publication on acceptance

- support for research data, including large and complex data types

- gold Open Access which fosters wider collaboration and increased citations

- maximum visibility for your research: over $100 \mathrm{M}$ website views per year

At $\mathrm{BMC}$, research is always in progress.

Learn more biomedcentral.com/submissions 